\title{
Towards Long-Term Social Child-Robot Interaction: Using Multi-Activity Switching to Engage Young Users
}

Alexandre Coninx ${ }^{1}$, Paul Baxter ${ }^{2}$, Elettra Oleari ${ }^{3}$, Sara Bellini ${ }^{3}$, Bert Bierman $^{4}$, Olivier Blanson Henkemans ${ }^{4}$, Lola Cañamero ${ }^{5}$, Piero Cosi $^{6}$, Valentin Enescu ${ }^{7}$, Raquel Ros Espinoza ${ }^{1}$, Antoine Hiolle ${ }^{5}$, Rémi Humbert $^{8}$, Bernd Kiefer ${ }^{9}$, Ivana Kruijff-Korbayová ${ }^{9}$, Rosemarijn Looije ${ }^{4}$, Marco Mosconi ${ }^{3}$, Mark Neerincx ${ }^{4}$, Giulio Paci ${ }^{6}$, Georgios Patsis ${ }^{7}$, Clara Pozzi ${ }^{3}$, Francesca Sacchitelli ${ }^{3}$, Hichem Sahli $^{7}$, Alberto Sanna ${ }^{3}$, Giacomo Sommavilla ${ }^{6}$, Fabio Tesser ${ }^{6}$, Yiannis Demiris ${ }^{1}$, Tony Belpaeme ${ }^{2}$

${ }^{1}$ Imperial College London, United Kingdom

2 The Cognition Institute, Plymouth University, United Kingdom

${ }^{3}$ Fondazione Centro San Raffaele, Milan, Italy

${ }^{4}$ Organization for Applied Scientific Research, The Netherlands

${ }^{5}$ University of Hertfordshire, United Kingdom

${ }^{6}$ National Research Council - ISTC, Italy

${ }^{7}$ Vrije Universiteit Brussel, Belgium

${ }^{8}$ Aldebaran Robotics, Paris, France

${ }^{9}$ Deutsches Forschungszentrum für Künstliche Intelligenz, Germany

Social robots have the potential to provide support in a number of practical domains, such as learning and behaviour change. This potential is particularly relevant for children, who have proven receptive to interactions with social robots. To reach learning and therapeutic goals, a number of issues need to be investigated, notably the design of an effective child-robot interaction (cHRI) to ensure the child remains engaged in the relationship and that educational goals are met. Typically, current cHRI research experiments focus on a single type of interaction activity (e.g. a game). However, these can suffer from a lack of adaptation to the child, or from an increasingly repetitive nature of the activity and interaction. In this paper, we motivate and propose a practicable solution to this issue: an adaptive robot able to switch between multiple activities within single interactions. We describe a system that embodies this idea, and present a case study in which diabetic children collaboratively learn with the robot about various aspects of managing their condition. We demonstrate the ability of our system to induce a varied interaction and show the potential of this approach both as an educational tool and as a research method for long-term cHRI.

Keywords: Case study, child-robot interaction, integrated system, knowledge gain, long-term interaction, multi-objective support, multiple activities, motivation

\section{Introduction}

Human-robot interaction (HRI) research seeks to understand the interaction between humans and robots and build systems to support that interaction. As research on robotic systems design and embodied artificial intelligence progresses, robots gain increasingly advanced sensorimotor abilities, 
cognitive skills, autonomy and safety of use. The rapid development of humanoid robots in recent years has also kindled a new interest for social robotics in the research community and for the general public alike. It has therefore become an important research topic to define and understand what constitutes a successful human-robot interaction experience (specifically for children, as our target user group). We also need to study the underlying social, cognitive and relational mechanisms in order to apply those principles to the design of new robotic systems that can achieve useful tasks and hold various roles in a real-world environment that includes humans.

Our domain of interest is the development and application of robotic companions to aid children with diabetes to learn how to manage their condition as they become increasingly independent (Blanson-Henkemans et al., 2012) (Fig. 1). This problem requires the robot to support behaviour and habit change (over extended periods of time), to facilitate learning of facts and concepts (over shorter time scales), and to provide this in a manner conducive to positive child temperaments. A key concept in attempting to achieve these goals is motivation: The child interacting with the robot must be motivated to achieve both short and long-term objectives. Whether intrinsic, extrinsic or both (Ryan \& Deci, 2000), motivation is required for learning (Keller, 1987; Christophel, 1990). Indeed, following a recent model of personal guidance systems for health support (Nalin, Verga, Sanna, \& Saranummi, 2013), motivation is a key component to support achievement of goals over the longer term too.

In any such application, the robot must therefore, to the greatest extent possible, support this motivation. The self-determination theory, for example, suggests that a sense of security and relatedness in an interaction will tend to lead to a greater motivation support (Ryan \& Deci, 2000). Evidence also supports the view that adaptive motivational patterns promote personal achievement goals (Dweck, 1986), with personal interest playing an important role (Schiefele, 1991). Previous studies with robots have shown that the interaction quality drops quickly when the robot's behavioural repertoire is too limited, as indicated by Kanda, Hirano, and Eaton (2004) and Tanaka, Cicourel, and Movellan (2007), and that the children become bored if the interaction is too repetitive and predictable, as suggested by (Ros, Baroni, \& Demiris, 2014). Having a robot whose behaviour is readily stereotyped and/or repetitive is therefore unlikely to fulfil the long-term requirements for social robots. Increasing the personal investment of an individual towards a learning goal by robot adaptation through personalised social interactions therefore seem to be important strategies that should be leveraged, and which appear to be particularly relevant to our application context.

In this paper, we seek to motivate, describe and validate our approach that is based on this perspective. We consider not only adaptation of behaviour, but also personalisation of the interaction structure itself with respect to the learning objectives as a means of supporting motivation, and hence learning and behaviour change, over extended periods of time. From this, we propose interaction structure adaptation by multi-activity switching within single interactions by a personally adaptive social robot. First we establish the basis of our approach in principle, and then present a novel demonstrator system that seeks to exploit this proposed solution. Finally, we conduct a case study to provide a practical proof-of-concept in a real-world application domain, which validates our work as the basis for a promising future approach to social child-robot interaction with learning objectives.

\footnotetext{
Authors retain copyright and grant the Journal of Human-Robot Interaction right of first publication with the work simultaneously licensed under a Creative Commons Attribution License that allows others to share the work with an acknowledgement of the work's authorship and initial publication in this journal.
}

Journal of Human-Robot Interaction, Vol. 5, No. 1, 2016, Pages 32-67. DOI 10.5898/JHRI.5.1.Coninx 


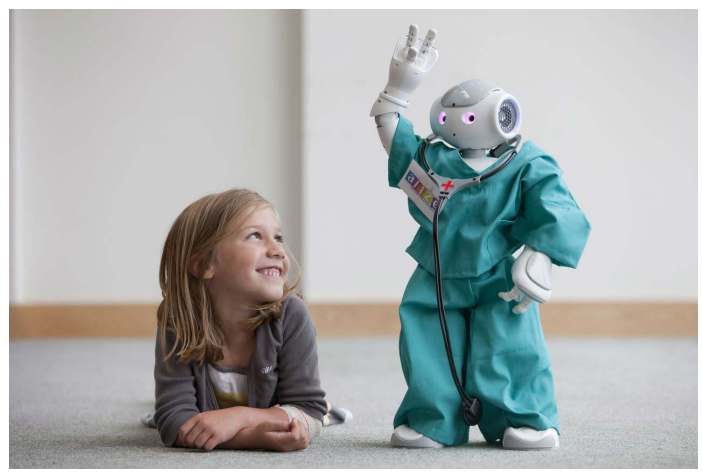

Figure 1. Our application domain is diabetes-related education through play in a hospital environment using robotic companions: this raises multiple issues of social interaction, expectation management and continued engagement that we seek to address through multi-activity interactions. In this work, we use the Nao humanoid robot (Aldebaran Robotics), shown above.

There are numerous further challenges involved in attaining such practically useful robotic systems. In the most general sense, these encompass problems such as technical development, taking into consideration the requirements of long-term interaction with humans and robots, with the specific requirements and sensitivities of having robots interact with children.

Firstly, the development of advanced robotic platforms where the multiple technologies that allow for rich human-robot interaction (sensory processing, verbal and non-verbal communication, cognitive abilities, etc.) are required. These systems have to be flexible enough to allow the exploration of various interaction situations, and robust enough to sustain extensive interaction with naive experimental subjects (Dautenhahn, 2007; Leite, Martinho, \& Paiva, 2013). In the exploration of social interactions, many previous HRI studies use "Wizard of Oz" (WoZ) methodologies, where all or part of the robot behaviour is remote-controlled by a human operator, to circumvent some of these open technical issues (Riek, 2012). This allows some studies that would have been impossible otherwise (or considerably more expensive) given the current state of the art in autonomous robotic technology, but the impact of the use of a WoZ has to be assessed and controlled. More generally, we strive to make interactive robotic systems as autonomous as possible. The WoZ is gradually phased out in an iterative manner (Kelley, 1984) as the robot's autonomous competencies increase and our understanding of autonomous human-robot interaction grows. This drive to incorporate autonomous operation for social robots is necessary given the high and continuous workload required from WoZ techniques, and given the highly variable and unstructured human-centred environments in which such social robots are intended to operate.

Secondly, building robotic systems able to engage in interactions with children also raises specific issues. Child-Robot Interaction (cHRI) is different from adult HRI in numerous ways, notably due to the ease with which children anthropomorphise robots and treat them as social agents rather than as machines (Belpaeme et al., 2013). In some ways this makes it easier to establish and maintain social relationships between children and robots, but it also means that children have strong expectations for robots to produce appropriate and timely responses to the users' actions and to process and use sensory information in socially sensible ways. These requirements make it more difficult to experiment with fully autonomous systems and often dictate the use of partial WoZ methodologies to make up for technical shortfalls. Care must also be taken to design every aspect of the experience 
around the child-robot interaction (including details such as briefing and debriefing) and to handle interaction breakdowns in a way consistent with children's expectations (Salter, Werry, \& Michaud, 2008; Ros et al., 2011). These requirements increase the system's integrative complexity and the amount of work necessary to prepare and run experiments.

In addition to these challenges, the overall purpose of robotic systems requires consideration. Even if our primary research topic is the interaction between human and robot itself, that interaction always takes place in a specific context and typically to achieve a specific user-directed goal, as is the case in the present study. If we seek to build robotic systems that are relevant and useful to humans in real-world social situations, that goal has to be more than a mere excuse to make a human and a robot interact together. For example, if in an educational context, then efficacy of the robot system in facilitating learning should be taken into account. If in a medical setting, then perhaps stress reduction would be a primary concern (e.g. Csala, Németh, \& Zainkó, 2012). However, it is probable that multiple objectives will need to be fulfilled simultaneously, possibly requiring tradeoffs between them. It is therefore unlikely that performance in a single or specific task would be a sufficient metric for evaluation. The design principles behind such a system should therefore be related to the ability of the robot to facilitate one or several higher-level goals (such as increasing the child's knowledge). The system should be evaluated with regard to these higher level goals.

We propose a robotic system that makes use of several activities and uses them jointly, as building blocks from which we may support a deep and varied child-robot interaction by reducing stereotyped and repetitive interactions, promoting the possibility of personalising both structure and content of these interactions. Taking advantage of the facets of modular, component-based control software architecture, we can integrate behavioural modules and underlying robotic technologies in a robust platform that facilitates longer-term interaction, make use of both autonomous and wizarded control in a seamless way, and provide a rich, coherent experience oriented towards desirable userdirected goals. In doing so, we extend upon our previous work (e.g. Kruijff-Korbayova et al., 2012, 2014), by detailing the fundamental framework upon which the work is based, significantly extending the technical system, and providing a real implementation to our application domain according to previously elaborated motivations (Baroni et al., 2014).

The purpose of this paper is to motivate and describe this approach in detail and to validate it through a case study for the child diabetes support application in a hospital environment, as described above. We then show how motivation can be bolstered to facilitate longer term interactions that can bring about learning and behaviour change. First we lay out existing approaches and systems in more detail, thereby identifying the scope for our novel approach to facilitate the maintenance of motivation (section 2). On this basis, we construct the specific multi-activity perspective we take in our research efforts (section 3), and then describe the system that has been developed on this basis (section 4). In order to validate the general approach, we then present a case study with a number of diabetic children (section 5), thereby demonstrating the utility and potential of the approach.

\section{Background}

Child-robot interaction has received significant attention from the HRI research community in the last decade, due on one hand to the interest raised by the specificities of interaction in child-robot dyads, and on the other hand to the vast potential applications in the fields of education, healthcare and entertainment (e.g. Ros et al., 2011). Given the relatively initial technological state-of-the-art in this regard, a great deal of research is focused on exploring how interactions between robots and children take place, and how the robot can and/or should behave in order to facilitate naturalistic interactions (as evidenced by qualitative self-report metrics, or objective behavioural measures). The efforts we present in this paper are similarly oriented: We seek to extend the effectiveness of child-robot interactions (in terms of quality and desired outcomes) by improving the behaviour of 
the robot. In this section, we provide an overview of existing work related to child-robot interaction; by so doing, we seek to motivate and justify the multi-activity perspective taken in our research.

We consider three aspects. Firstly, the exploration of behavioural and physical competencies of robots is reviewed, specifically regarding their influence on maintaining an interaction and interest in the robot. Secondly, we consider the strategies and effectiveness of various robot systems in terms of desired interaction outcomes (for example, educational or therapeutic goals). Finally, we review some general principles specifically related to longer-term interactions, which can pose some unique issues beyond those already prevalent in short-term interactions. Given our target application, the focus in each of these three points is specifically on studies that involve children, where the relevant studies have been conducted. Whilst this excludes numerous studies conducted in a similar domain but with adults, the particular requirements of dealing with children entail a more restricted focus (Salter, Werry, \& Michaud, 2007; Beran, Ramirez-Serrano, Kuzyk, Fior, \& Nugent, 2011; Belpaeme et al., 2013).

We make the implicit claim through our work that real physical robots confer an advantage in HRI outcomes over virtual agents (e.g. avatars, etc.). Since this is not at present an uncontroversial assertion, we first briefly consider and justify the role of robot embodiment itself. A range of efforts have focused on the use of simulated or virtual agents and avatars, in addition to physically embodied robotic devices, with some efforts to combine the two (Segura, Kriegel, Aylett, Deshmukh, \& Cramer, 2012). In addition to the clear advantage that physical robots have in terms of real world interaction and manipulation (Stiehl et al., 2009; Shibata, 2011), regarding the difference between the embodiment types, there are three aspects that indicate some (potential) advantage is conferred by the presence of a physical robot. Regarding user perceptions, there are a number of studies that have shown that a physical robot is more appealing (Wainer, Feil-Seifer, Shell, \& Mataric, 2007; Komatsu \& Abe, 2008) with greater social presence (Lee, Jung, Kim, \& Kim, 2006), but that this may lead to higher expectations of a potential interaction (Lee et al., 2006). Regarding differences in interaction behaviour (from the perspective of the human), there are a number of results that indicate the non-conscious effect of a physical robot presence: for example, Looije, van der Zalm, Neerincx, and Beun (2012) and Kennedy, Baxter, and Belpaeme (2015) have shown that children will gaze more often and longer at a real robot than a virtual version of the same robot. In terms of improved outcomes resulting from using physically embodied robots as opposed to virtual agents, there is some evidence of a benefit to performance or other outcome when using real robots, as shown in the context of learning (Bartneck, 2003; Leyzberg, Spaulding, Toneva, \& Scassellati, 2012), long-term behaviour change (Kidd \& Breazeal, 2008), and motor skills (Kose-Bagci, Ferrari, Dautenhahn, Syrdal, \& Nehaniv, 2009). Taken together and applied to the present study, we therefore seek to take advantage of the physical embodiment of the robot, in addition to the developments in robot behaviour and interaction context that we introduce in this paper.

There are a number of (social) robots characteristics that encourage interaction from the perspective of children. Further to the robot's embodiment (as discussed above), the appearance of the embodiment itself can imply cognitive abilities (Turkle, Breazeal, Dasté, \& Scassellati, 2006; Hyun \& Son, 2010; Beran et al., 2011), thus extending the implied possible interaction beyond that typically achievable with toys. Whether related to this effect directly or not, there is some indication that children will comply with instructions or suggestions from a robot (Kennedy, Baxter, \& Belpaeme, 2014), and also tend to align their behaviour with that of the robot (Nalin, Baroni, Kruijff-Korbayova, et al., 2012) during an interaction. Overt behavioural cues from the robot, such as gestures (Sidner, Lee, Kidd, Lesh, \& Rich, 2005) and gaze (Markus, Eichberg, \& Andre, 2012) are necessary to enable interaction; these tools can even be utilised with simplified embodiments (Kozima \& Nakagawa, 2006). Furthermore, the presence of unexpected behaviour (as perceived by the child, whether intended or not) appears to be a factor in helping to maintain attention through- 
out the interaction (Salter et al., 2008). Affective communication has also been shown to provide additional facets to robot behaviour, making the relationship between robot and child deeper and richer. For example, emotional pose recognition with the Nao robot is consistent (Beck, Canamero, \& Bard, 2010), facilitating the use of emotional expression as a feedback channel that can be employed by the robot. Following from this general competence, it has been shown that an empathic or supportive robot is prefered to a non-affective agent by children, and that its use can possibly lead to increased performance, in both teaching scenarios (Saerbeck, Schut, Bartneck, \& Janse, 2010), and peer-peer interactions (Leite, Castellano, Pereira, Martinho, \& Paiva, 2012). Thus, what we see is that there are a range of physical and behavioural competencies, typically inspired directly by human behaviour, that are employed to make robot behaviour more naturalistic within a child-robot interaction context.

Given these robot characteristics that can be taken advantage of in child-robot interactions, increasing numbers of studies have assessed how such social robots can achieve some outcome with children, such as in therapy (Salter et al., 2007; Csala et al., 2012; Thill, Pop, Belpaeme, Ziemke, \& Vanderborght, 2012), interrogation (Wood et al., 2013), or entertainment (Billard, 2002). The typical application, though, is to assist the child in learning about some topic. For example, it has been proposed that robots could help diabetic children learn how to manage their condition (Belpaeme et al., 2012; Baroni et al., 2014). A number of approaches have been taken to help children with related skills: For example, independent maths and quiz games have been used (Janssen, van der Wal, Neerincx, \& Looije, 2011; Blanson Henkemans et al., 2013) to demonstrate the role of personalised robot behaviour on performance. In terms of teaching children more generally, robot tutors have compared favourably with human teachers in terms of attention paid to them (Draper \& Clayton, 1992), and robots with a more assistive role have been shown to be preferred (Short et al., 2014). What characterises these studies is that they focus on a specific task context, and within that, a single type of robot behaviour (notwithstanding some degree of personalisation or adaptation). The motivation to maintain interactions in support of some learning objective may become an issue over longer time scales and during repetitive game sequences (Ros, Baroni, \& Demiris, 2014) but this issue is not typically raised in these studies-although boredom is at times cited as problem for neurotypical children (Kozima \& Nakagawa, 2006).

The topic of long-term interaction between human and robots has been studied in several research projects, a recent overview of which can be found in Leite et al. (2013). A number of works have been focused on long-term adult-robot interactions, which emphasise the necessity for personalisation and adaptation, (e.g. Dautenhahn, 2004; Kidd \& Breazeal, 2008): Our focus here however is on long term interactions with children. In a study conducted with children of various ages over a two week period, it was found that children interacted less with a robot (given free choice) as time went on (Kanda et al., 2004). A further longer-term study found that by gradually unlocking different behaviours as a function of interaction time, this effect could be overcome, but only if the children considered the robot to be a peer (Kanda, Sato, Saiwaki, \& Ishiguro, 2007). These findings concur with the notion introduced above of a necessity for variation and novelty in an interaction to prevent predictability and boredom. This is related to the more general issue of motivation: facilitating, for example, continued engagement through enjoyable interactions (Dautenhahn, 2007). In terms of presence, using animated agents, with robots as one particular type of these, can encourage children to maintain engagement with a nutritional diary-filing exercise (Lu, Baranowski, Islam, \& Baranowski, 2012). With personalised goals, further motivation for interaction may be achieved (e.g. Janssen et al., 2011). Finally, motivating behaviours of the robot itself, following from the affective behaviours described above, can further facilitate this effect (Saerbeck et al., 2010).

In summary, we note that typical studies on the characteristics of robots, and indeed more finegrained analyses of the influence of their behaviours on interactions, result from short-term studies 
using highly structured and constrained interactions. When extended to longer term interactions, the importance of motivation to interact takes on even greater importance, and a stereotypical, repetitive robot behaviour can induce a progressive loss of interest from the subject. Considering desired outcomes for interaction, while novelty can provide a strong motivator for initial interactions, the ability of the robot to adopt a personalized behaviour becomes an increasingly important aspect in maintaining engagement within interactions. Our adaptive multi-activity approach seeks to take advantage of this effect to address the issue of longer-term motivation.

\section{Challenges and goals}

Using social robots to support children achieve some goal is an endeavour that both offers rich opportunities and poses specific restrictions for cHRI research. Building a system supporting multiple activities also raises a number of issues on its own as compared to one limited to a single type of interaction. In this section, we will first describe the specific problem domain for which our system is designed (section 3.1), then we move on to the underlying scientific and technical problems that need to be addressed (section 3.2), and finally we discuss the challenge of how to extract information from a heterogeneous, multi-activity interaction and measure the actual impact of the proposed system (section 3.3).

\subsection{The problem domain}

As part of the EU ALIZ-E project ${ }^{1}$, we investigate the potential of a multi-activity system as an edutainment tool for diabetic children receiving care in a hospital. Diabetic children have to learn a specific knowledge set to help them manage their disease. This includes recommendations about their diet and physical activity, as well as information about the medical treatment of diabetes (insulin injections, glycaemic tests, etc.). They also need support and entertainment during hospital stays that can otherwise feel long, boring and stressful. Animal assisted therapy has successfully been used in this regard (Fine, 2010), and there is hope that robot-assisted therapy can be used in the same way (Belpaeme et al., 2012; Nalin, Baroni, Sanna, \& Pozzi, 2012). The problem our system attempts to address is therefore twofold: reinforcing the children's knowledge and enhancing their wellbeing in the hospital.

This specific problem and the hospital environment in general have important strengths for HRI research. First, although the hospital can not been considered a lab environment, it is a more controlled environment than most other contexts where field cHRI experiments have previously been conducted (e.g. classrooms). Furthermore, the medical staff members are typically open to experimental support methodologies that may increase their patients' wellbeing. However, given the sensitive nature of the hospital environment, there are some limitations and regulatory constraints in terms of data collection and protection that increase the operational complexity (Ros et al., 2011). Finally, the fact that diabetic children have to visit the hospital regularly during a span of several months provides us with a setting that facilitates the study of long-term cHRI in a semi-structured manner.

\subsection{Designing a rich user experience with multiple activities}

From the perspective of the robot, various activities may have different requirements concerning the positioning and movement of the robot relative to its immediate environment, the human user, and possibly the external support devices (screen, control device, etc.) that are used in the course of the activity. For example, an activity focused on motion will need the robot to stand away from the user and to keep clear of obstacles. On the other hand, an activity where the robot plays a video

${ }^{1}$ URL: http://www.aliz-e.org/ 
game with the child will need both the robot and the user to remain close to each other and at arm's length of the gaming device. The robot therefore needs to demonstrate an advanced awareness of its environment by moving between different activity zones, changing postures, and dynamically keeping its position adapted to the nature of the activity without disrupting the social interaction. The variety of social situations and physical layouts including human and robot that can arise from this more complex interaction also makes collecting experimental data (such as video or depth image information) about the human-robot interaction more difficult.

From the perspective of the child, interacting with a single robot in an individual interaction session, but whilst performing multiple different activities, would give rise to a number of expectations that need to be managed. The robot's appearance of maintaining a consistent behavioural profile throughout the activity interactions is important, so that the child perceives the robot as a single agent, even if the robot control system is fractured at the activity level — which may be seen as being related to the robot's personality. This requires that any knowledge gained by the robot about the child through interaction (such as preferences, task performance, etc) is subsequently used in an appropriate and consistent manner. For example, if the robot is made aware of a child's favourite food in one activity, this information should be taken advantage of in future activities. Whilst superficially this is not necessarily a technical difficulty, the appropriate application of this functionality in a social context remains a challenge, with as of yet only limited work in adaptive robot personality in HRI (e.g. Tapus, Tapus, \& Matarić, 2008).

\subsection{Experimental validation of the system}

Designing a large system comprised of multiple activities allow us to investigate longer, more complex types of interactions that get closer to the behaviour of robot as educators, carers or general purpose companions, but measuring and evaluating those richer interactions poses new challenges. Even for smaller systems focusing on one type of specific interaction, designing methods and metrics to evaluate interaction quality can be made difficult by the constraints of interaction with children outside of a lab setting (Tanaka, Movellan, Fortenberry, \& Aisaka, 2006; Ros et al., 2011). The use of a more complex system where multiple activities are jointly used to achieve high-level goals make this issue all the more relevant. We must therefore strive to assess the quality of the interaction using general measurements, both subjective (questionnaires, interviews, etc.) and objective (data derived from the child behaviour), that focus on how well those objectives are met independently of each activity's specifics.

\section{The multi-activity robot system}

Having introduced the need for a multi-activity based approach to child-robot interaction to support longer-term motivation, we continue in this section by describing the system developed to embody the principles introduced, which constitutes a novel contribution to the state of the art in its own right. There are a number of aspects to this system that require detailing: The overall context that is provided for the interactions is described first (section 4.1), followed by a note on the importance of, and means of maintaining, coherent robot behaviour (section 4.2). We then describe the three constituent activities (section 4.3) and introduce some central aspects of the underlying technologies employed to support the partially autonomous behaviour (section 4.4).

\subsection{The system and its context: The robot theatre}

When developing a system to be involved in user evaluations, considerations of setup consistency for reproducibility are important. In order to address this, we put forward the concept of the robot theatre (Fig. 2). The robot theatre is a technical specification precisely defining the physical layout of the environment in which the child and the robot interact. It specifies the dimensions of the 


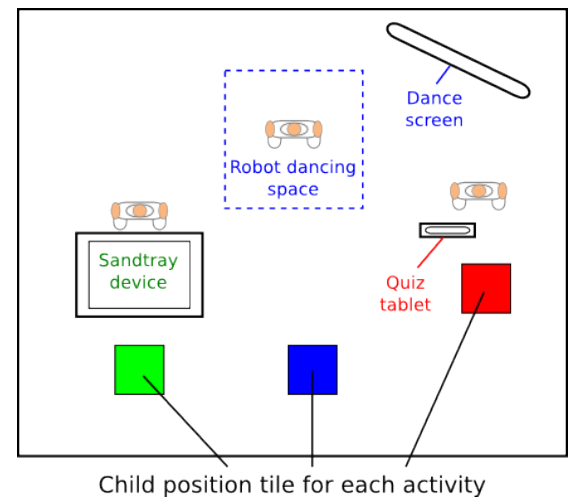

(a) Robot theatre schema

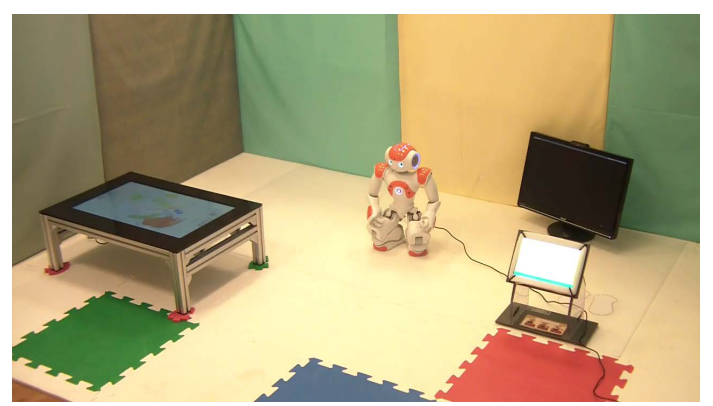

(b) Picture of the system used during experiments.

Figure 2. The robot theatre setup used during the experiments. Fig. 2a describes the position of the various support devices used for the three activities, and the position of the robot when playing each activity (activity zones). Fig. $2 b$ shows the experiment space: The child was instructed to go to the green tile when playing the Collaborative Sorting activity, the blue tile when playing Dance and the red tile when playing Quiz. The precise specifications of the physical environment were detailed in a technical document and used throughout the development and use of the integrated system by various project partners.

space (limits of which are materialized by partition walls and a special ground tiling), the starting position of the child and the robot, and the layout of the supporting devices required by the activities. This common environment allows us to integrate data from various experiments and data collection sessions. The specificities of each experiment, such as the locations of data collection devices (video cameras, Kinect sensors, microphones, etc.) and the details of physical behaviour of the child and the robot during activities can be defined relative to robot theatre's general framework, thus facilitating consistency and replicability.

The robot theatre was built around the three activities of our multi-activity system: turn-taking quiz, creative dance and collaborative sorting. The physical layout of the theatre included visual cues about where the child was expected to stand while doing each activity (see Fig. 2b). It was also designed so to allow for easy movement between activities for both child and robot, and to allow the robot to keep a coherent and seamless behaviour while interacting with the child during and across activities.

\subsection{Coherent Robot Behaviour}

In having a system with multiple different activities, it is important for the robot to maintain a consistent and coherent behaviour between them. This ensures that the child interacting with the system perceives interacting with a single agent and not with one with multiple personalities, thus supporting and improving the possibility of longer term engagement and interactions. The 'games console' approach to interaction and behaviour design is therefore to be avoided: Instead of considering each of the activities as stand-alone and largely independent 'apps' to be run, care has been taken to ensure all levels of robot behaviour, from perceived personality to behavioural expression, remain consistent throughout all activities. The role of the activities thus goes beyond the provision of mere 'content' for the robot theatre, since the specific role of the robot within the activities, and indeed the specific role of each of the activities for the wider goal (section 4.3), needs to be taken into account. 


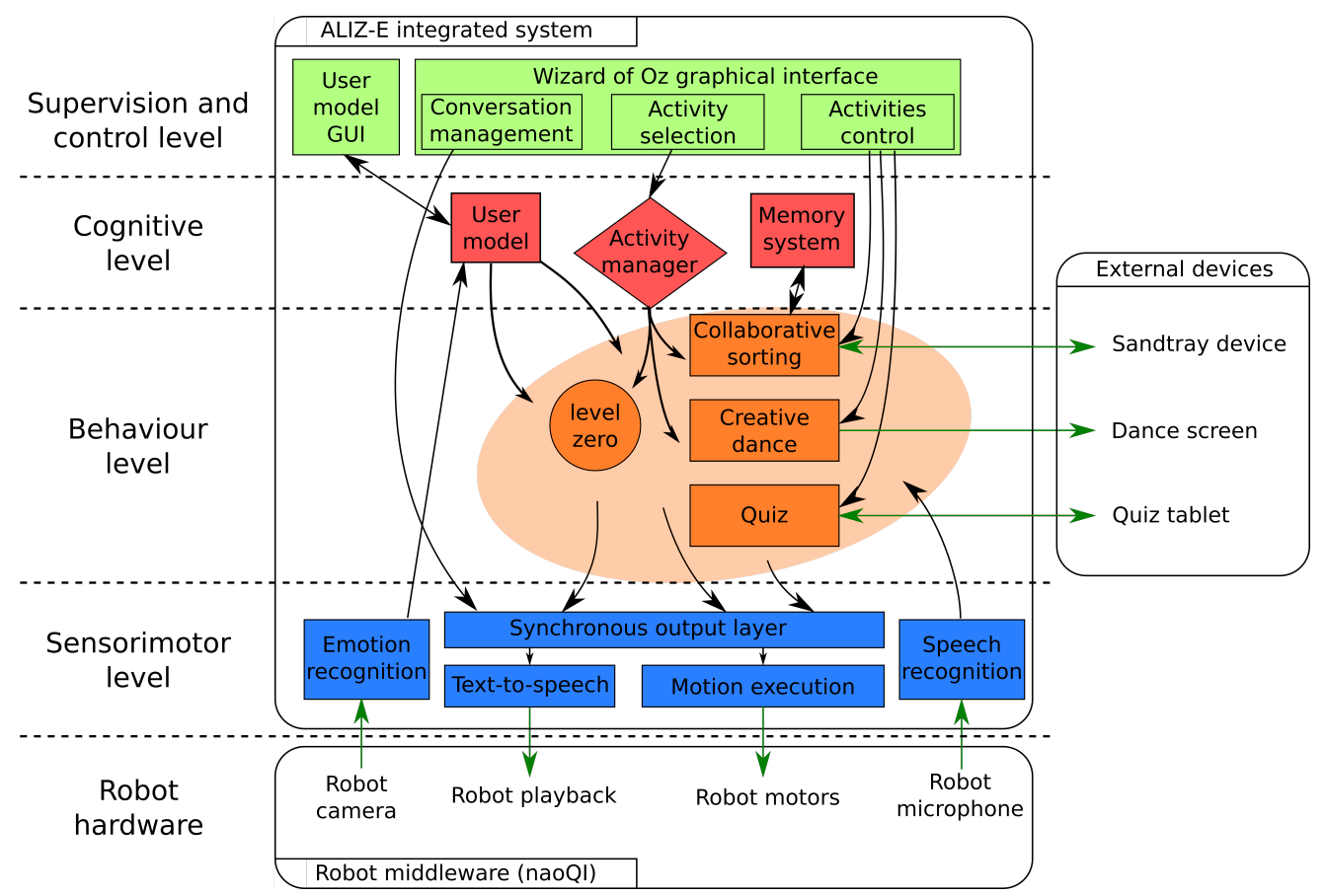

Figure 3. Schematic description of the integrated system architecture. Black arrows depict Urbi message exchanges (see section 4.4), while green arrows show interaction with the robot's middleware or external devices. The low-level motor components are shown in blue, the activities that determine the robot behaviour in orange, the intermediate cognitive structures (memory, behaviour selection, etc.) in red, and the top-level wizard-based control system in green.

To achieve this, we employ a number of strategies that are instantiated in the robot's fundamental control architecture (Fig. 3). Firstly, a basic behavioural layer ('level zero') is implemented that is responsible for the general behaviour of the robot, such as life-like movements (small motor movements, particularly on the upper body of the Nao robot, blinking eyes, etc.). This behaviour is always activated and persists within and between all of the activities (Fig. 3). So, even when the robot is not explicitly engaged in one of the activities, it still fundamentally behaves in a similar manner. It also handles robot positioning in the experimental space (Fig. 2) using a specific behavioural module that has the child walk the robot by holding its hand and guiding it towards the right position, which both supports bonding between child and robot and provides a solution to the problem of having the robot navigate its environment to reach the appropriate position for each activity.

Secondly, the child's data (name, age, preferences, performance, etc.) is stored in a central repository (the 'user model') that is accessible by each of the activity controllers. Additionally, emotional state may be stored here (valence and arousal, as automatically detected if possible, and as set by the wizard if not). This ensures that something learned during one activity about the child is available to other activities, which again supports behavioural consistency and moves away from the 'games console' perspective.

Thirdly, we have implemented a wizard-controlled ${ }^{2}$ conversation management functionality that

\footnotetext{
${ }^{2}$ This is done for technical reasons at this stage: There is no reason in principle why this functionality could not be
} 
operates within and between each of the activities. Having this functionality outside of the control of an individual activity ensures that the qualitative nature of conversation events remains consistent across the activities. The conversation management system is used to provide three different types of child-robot verbal interaction:

- Activity and session management talk: the discussion that arises from the general structure of the multi-activity interaction. This includes explanations given by the robot to the child about the activities, discussion about activity switching, requests from the robot to be helped to move around in the robot theatre, and the graceful handling of failure modes (if the robot falls, runs into low battery, etc.);

- Small talk: salutations and general discussion about superficial topics to establish and maintain the child-robot social bond (Blanson Henkemans et al., 2013);

- Off-activity talk: informal discussion about topics that arise from the activity content and are relevant to the overall goal of the interaction (e.g. in our case, talk about diabetes and its management, which can both increase the child's interest for the robot and support the therapeutic goals of the interaction; Kruijff-Korbayova et al., 2014).

Fourthly, a component that supports the non-verbal behavioural adaptation of the robot (the 'memory system') has been added: This helps to ensure that a similar adaptation occurs in the activities. Finally, a central 'activity manager' component is responsible for starting, stopping and switching between the different activities, and pausing/resuming within an activity, in a smooth manner (Fig. 3). It ensures that activity transitions do not cause interruptions to the interaction by preventing inconsistent robot behaviour changes.

Finally, a thread that runs through each aspect of the system, and which thus contributes to the coherency of the robot's behaviour, is the role that adaptivity plays. We distinguish between two targets of adaptation. The first is concerned with altering various aspects of the robot's knowledge and systems to adapt to aspects of the child. For example, the child's performance may be used to elicit different robot behaviours or strategies, and the child's name and personal preferences may be used in the conversation management system. This type of adaptation is implemented at the level of individual components (described below in section 4.4). The second target of adaptation is the personalisation of the structure of the interaction itself, which forms the focus of the present paper. Introduced above, this is the possibility for the child to express preferences in deciding what activities to engage in with the robot.

Together, these five general implementation and behavioural strategies contribute to the robot appearing as a coherent single agent to the interacting child; further technical detail on each of these strategies may be found below in section 4.4.

\subsection{The activities}

The system we developed to instantiate the principles described above incorporates three distinct activities (Fig. 4). This facilitates an illustration of the utility of activity switching to support personalisation. This was formulated for our application domain; other types of activity, or the same activities with different contents, may be more suitable in other application domains.

Given our application context of supporting children's knowledge gain through entertaining interactions with a robot (section 3.1), we use the three activities to approach the diabetes-learning problem from different perspectives. While they all attempt to support the learning of the child on the same topic (diabetes self-management for our application), the three activities employ different means of engaging the child with material to be learnt. Therefore, whichever of the activities are chosen (and in whatever combination), the child is encouraged to learn. This also better enables

autonomous in the future, although this would require advancement in the current state of the art (see section 4.4). 


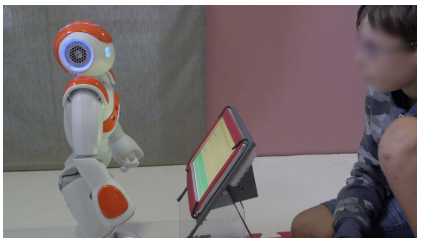

(a) Turn-Taking Quiz

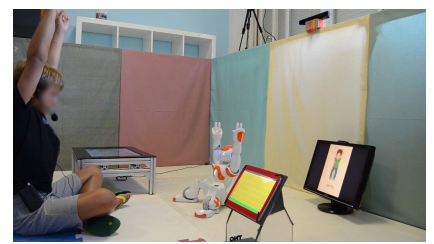

(b) Creative Dance

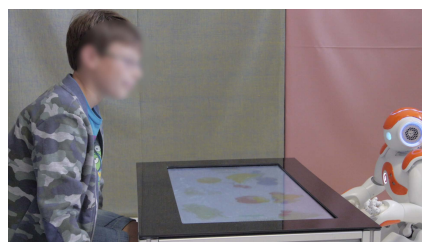

(c) Collaborative Sorting

Figure 4. Children engaged in each of the three activities in the robot theatre.

the robot to be sensitive to personal preferences and learning styles, given the different approaches available.

Each of these activities has a separate control system that handles the logical flow of events, the flow of information, and any specific behaviours that the robot should perform. In terms of system implementation this arrangement is necessary for practical reasons (interaction design, system stability, etc). However, for a multi-activity system, it is of utmost importance that the robot's behaviour remains coherent. As described above, we achieve this by having a low-level layer of common robot behaviours. In addition to this, the activity manager ensures that switching between activities (and behaviour outside of an activity) occurs smoothly from the child's perspective. To support this, each activity needs to be able to provide a graceful means of stopping, pausing and resuming its interactions. This provides a number of constraints on the operation of the activities as they operate on the principle that they could receive a request to stop or pause at any moment, and resume at some later point. The contents and characteristics of the three activities are as described below.

4.3.1 Turn-Taking Quiz is a multiple-choice quiz activity between the robot and the child (Fig. 4a), with two possible configurations: either the child can ask questions of the robot, or the robot can ask questions of the child. Both may occur within a single interaction. To support this interaction, a tablet device on a see-saw mechanism is used to display the questions and answers, which is oriented towards the party asking the questions (Fig. 4a). The questions are drawn from a predefined database formulated in collaboration with healthcare professionals and are on a range of topics relevant to diabetes and its management. This activity is primarily verbal based (KruijffKorbayova, Kiefer, Baroni, \& Zelati, 2013) and may be viewed as the most competitive of the three activities, with separate scores for the two participants (Blanson Henkemans et al., 2013).

4.3.2 Creative Dance is an activity that mostly focusses on the child performing some motions following instructions or demonstrations provided by the robot (Fig. 4b; Ros \& Demiris, 2013). It relies on direct interaction between the child and the robot but also involves a screen used as a support device that can be used to provide the child with extra information (such as images or further instructions). From a clinical perspective, the dance activity encourages the child to engage in physical activity, which is important for children with diabetes and also provides a framework in which information about nutrition, exercising and maintaining a healthy lifestyle can be shown to the children. From an HRI research perspective, the dance activity allows the collection of data about child-robot nonverbal communication, imitation and overall interaction (Tanaka et al., 2006; Ros, Baroni, \& Demiris, 2014; Ros, Coninx, et al., 2014). This activity is thus overtly physical and encourages learning about diabetes-related concepts through association with postures and movements. 
4.3.3 Collaborative Sorting is an activity centred around the Sandtray system (Fig. 4c) involving a touchscreen with which both the child and robot can interact and collaborate (Baxter, Wood, \& Belpaeme, 2012). The task is a sorting game with a communal score for both players, in which pictures of different food types have to be sorted into one of two categories: In this game, both the child and the robot can make moves on the screen (virtually in the case of the robot). Given the necessity for diabetic children to learn the carbohydrate (among other aspects) content of foods in order to determine insulin intake, we use a carbohydrate-content-sorting game to facilitate learning this. This is supplemented with sorting games based on healthy activities, food quality, and sanitary habits, which therefore also supports a wider range of diabetes-related knowledge in the same collaborative sorting context. The touchscreen provides visual and aural feedback to categorisation events for both child and robot moves, and the robot additionally makes sympathetic comments on the child's most recent categorisation attempt (e.g. "That was right, well done!" or "Too bad, why don't you try another one?"). Previous studies have shown that children interacting with one another in the context of the Sandtray (i.e. without the robot) demonstrate rich social behaviour (Kennedy, Baxter, \& Belpaeme, 2013), an effect we attempt to leverage in child-robot interactions (Baxter, Baroni, Nalin, Sanna, \& Belpaeme, 2013; Baxter, deGreeff, \& Belpaeme, 2013). In the context of the multi-activity system described here, the Sandtray provides the opportunity for physical interaction in collaboration with the robot and immediate feedback to the attempts made by either interactant (child or robot), which complements the approach taken by the two other activities.

\subsection{Supporting technologies}

Our integrated system architecture relies on the Urbi platform (Baillie, 2005; Baillie, Demaille, Hocquet, Nottale, \& Tardieu, 2008), which allows integration of various software components written in different languages (C++, Java) and running on heterogeneous systems (the robot itself and remote computers) using an event-based high-level orchestration language, UrbiScript.

The Nao robot itself-which included a full x86-compatible computer running GNU/Linux-is the central hub of our system. Software components directly related to robot hardware (motor control, sound playback, etc.), as well as those handling the general behaviour of the robot during and through activities, were implemented in UrbiScript directly on the robot. Other components, making use of external resources (remote control GUI, Kinect motion recognition, etc.) or demanding more computing power (speech recognition, image processing, etc.), were implemented in Java or C++ and run on remote computers. These remote components use the UObject API, which easily allows the component to connect to the Urbi platform on the robot through the wireless network and be directly accessible as an object in the UrbiScript execution flow (Fig. 5). This structure allows us to easily add new components providing new capabilities as they are developed, or to selectively disable some systems when they are unneeded or when required hardware is not available; this flexibility is one of the main factors that allowed us to build a coherent integrated system from software components coming out of several project partners with different expertise.

Our system is therefore composed of independent modules that communicate through Urbi events (Fig. 3), organized in a layered way.

Closest to the robotic hardware sit the sensorimotor components that directly control the robot's motor behaviour and voice output, while taking information from its sensors. The motion execution component generates motor orders and modulates them to achieve expressive motions, giving the robot a lifelike behaviour (Beck, Hiolle, \& Canamero, 2013; Hiolle, Lewis, \& Cañamero, 2014). The text-to-speech component, based on the MARY-TTS system, provides expressive voice synthesis and playback through the robot speakers (Tesser, Sommavilla, Paci, \& Cosi, 2013). Both motion execution and text-to-speech are controlled by the synchronous output module, which coordinates voice and motion output to produce composite verbal and non-verbal behaviours; this module also 


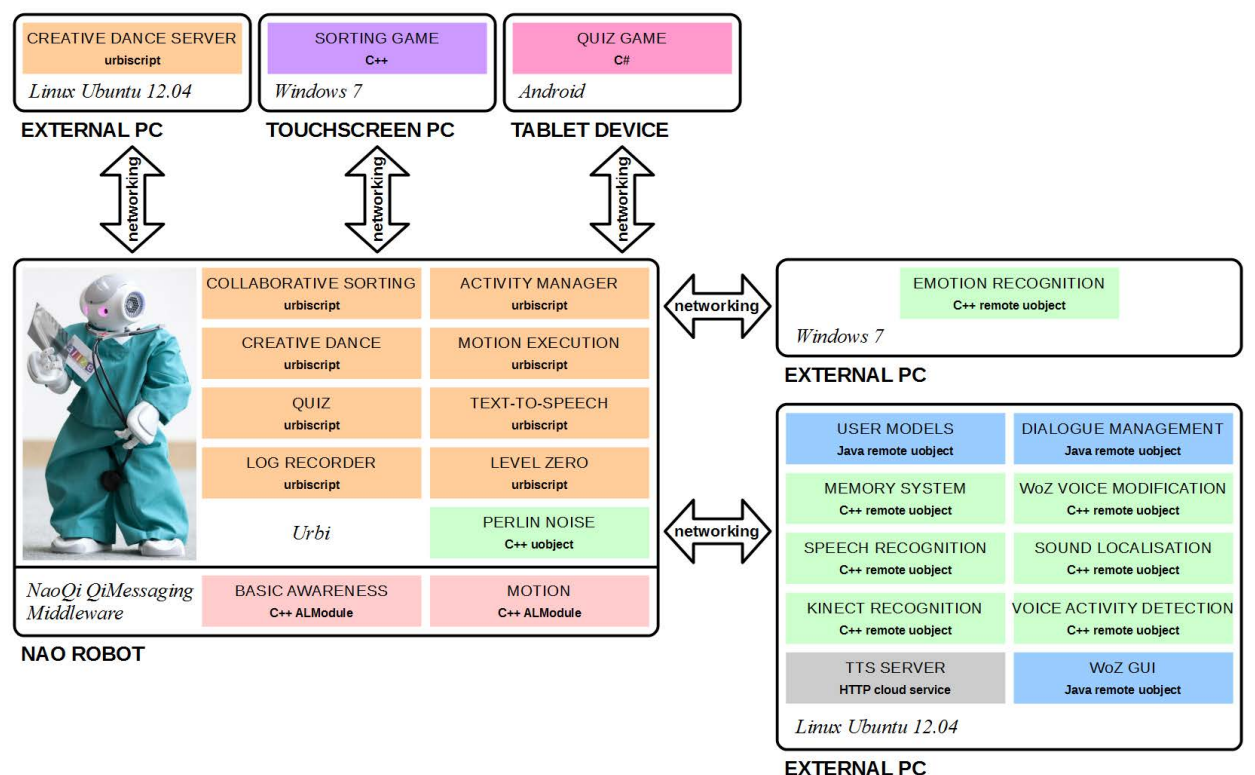

Figure 5. The multi activity system developed for the present work is comprised of 25 components (using five different programming languages) distributed over five external devices (using four different operating systems in a wireless LAN) in addition to the Nao robot. The UrbiScript middleware provides orchestration for the integrated system, with the wizard (WoZ) providing only top-level control. For a schematic control system overview, please refer to Fig. 3.

handles the sharing of motor resources through a queueing system. From a perceptual viewpoint, the speech recognition component performs voice detection and processing of the child's verbal input (Cosi et al., 2012), while the emotion recognition system process the camera output, performs face detection and provides an estimation of the child's emotional state based on facial expression (Wang, Enescu, \& Sahli, 2013).

It should be noted that while all the described sensory components operate continuously, in real time and in an autonomous way, the sensorimotor loop is not yet closed at every possible level and not all of the collected data is directly used to drive the interaction. Experimental sensory components such as speech recognition and emotion recognition tend not to be reliable enough yet to drive the interaction in weakly controlled experimental conditions like the setup we propose. The present contribution's primary focus-the study of high-level, long-term social interaction between child and robot-requires us to have a smooth and efficient interaction and overrides the objective to build a mostly autonomous system. We therefore choose to place a significant part of the robot's behaviour under the supervision of a human operator (wizard), who has access to the output of the sensory components but ultimately makes decisions based on both that data and his perception of the scene as mediated by audio and video streams captured by recording equipment. More specifically, the voice detection, speech recognition and emotion recognition systems are used in that manner, to assist the wizard and to collect data to be analysed offline, but do not directly influence the motor components. We will describe the operator's role more closely in section 5.2.1.

The higher, behavioural level is comprised of the activities that define a specific interaction framework between the robot and child. Additionally to the three activities described in section 4.3, 
the level zero behaviour is a baseline activity that simply gives the robot a basic lifelike behaviour when not engaged with other activities. The activity components control robot output though the synchronous output layer, and interact with the external support devices used by activities.

The above cognitive level is concerned with the memory, learning and activity selection functions. Those features, described in section 4.2, are common to all activities and are those that ensure a coherent robot behaviour, independently of the specific interaction framework defined by activities.

At the top level is the supervision Graphical User Interface (GUI) that is presented to the operator in the context of the Wizard of Oz method. The wizard has three main roles. First, it controls the starting, pausing and stopping of the successive activities through the activity manager. Second, it controls the execution of the current activity through a dedicated interface specific to each activity. Finally, it can directly interface with the synchronous output layer to produce motion and speech output, for example, to talk with the child while the current activity is paused, or to reposition the robot between activities. A distinct GUI is connected specifically to the user model, allowing the display and manual modification of the information stored inside.

\section{Hospital-based Case Study}

5.1 Goals and context

After having motivated the use of multi-activity switching within interactions and the system that embodies this principle, we now evaluate through a case study whether such an approach can find meaningful application to our target domain. There are two aims for this case study. Firstly, we assess how the activity switching dynamics unfold, and how they are related to the children's behaviour. Secondly, we assess the potential of such an approach to support children with type I diabetes following the overall goal to manage their condition independently.

In collaboration with the medical professionals of Ospedale San Raffaele's paediatric ward (Milan, Italy), a protocol was formulated in order to cover both of these aspects of our study. Four desired learning outcomes were identified and linked to the overall goal of improving self-management of diabetes by the children:

1. Guidelines for a correct nutritional behaviour and healthy lifestyles;

2. The importance of recognizing the composition of foods in terms of carbohydrates, proteins and fats;

3. Discrimination between high/low-carbohydrate content foods;

4. Sanitary norms for a correct management of the therapy.

Following these goals, the content of the three activities (creative dance, collaborative sorting and turn-taking quiz) were modified to encompass these topics in different ways (Fig. 6). As a result, the intention is that the interacting child can learn about one or more of the desired learning outcomes in whichever activity is chosen, with a different learning approach employed by each of the activities (see Fig. 6 and section 5.2.3).

The study was conducted in the summer of 2014 at the Ospedale San Raffaele facility. All data collection (questionnaires, interviews and recorded speech) was therefore conducted in Italian, with the results presented here their translated versions.

\subsection{Methodology}

5.2.1 Study design and procedure Each participant engaged in three interactions with the multiactivity robot system in the context of the robot theatre over a period of between one and two months. Each interaction lasted, at most, one hour. All children started their first interaction with the same initial state of the system, apart from the prior provision of their name. In subsequent interactions, each subject interacted with the state of the system from the end of their previous interaction: all 


\section{Child progressed knowledge/habits state}

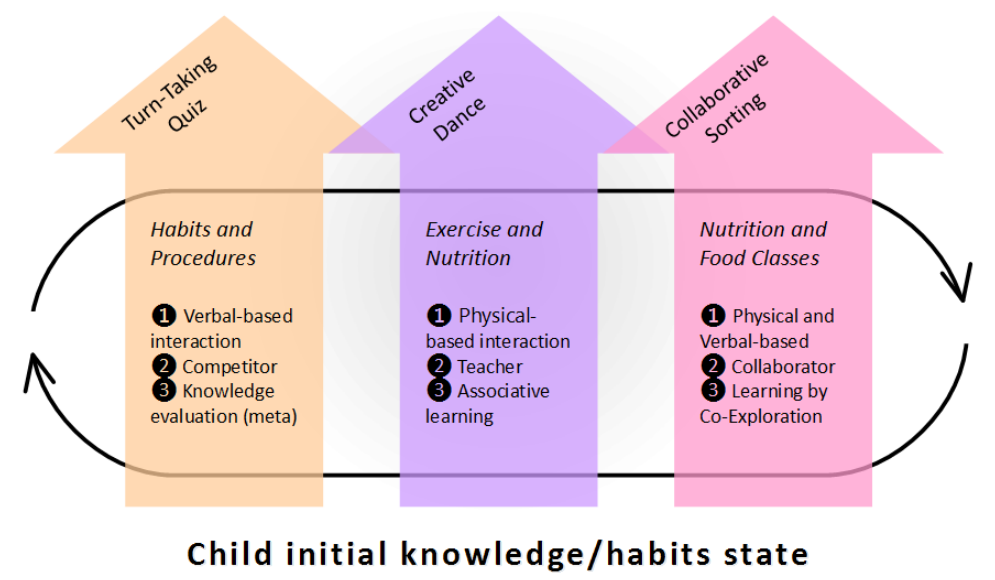

Figure 6. The three activities were designed with different interaction types (1), with the robot acting in different peer roles (2) and focused on leveraging different learning styles (3). The content of the activities was designed according to three central aspects of diabetes management. Given an initial state of knowledge and attitude of a child, the goal of the interactions was to facilitate the child's learning through engagement in a combination of activities that depended on the child' preferences.

adaptations and acquired data were thus maintained.

The non-autonomous aspects of the robot's behaviour were handled by an operator (the "wizard") who supervised the robot remotely, perceiving the interaction through robot sensors and video cameras positioned around the robot theatre. The operator was a technical expert and was assisted by a psychologist, especially to handle the off-activity talk component of the interaction.

At the beginning of each individual interaction, the child could choose the first activity. Afterwards, activity switching was handled by the operator following a set of rules (Fig. 7). These rules, and a full description of the wizard responsibilities, are described in section 5.2.3.

Prior to the first interaction, each subject completed a series of questionnaires, assessing both their state of knowledge in the interest areas (the Knowledge questionnaire), and their diabetesmanagement related habits (the Habits questionnaire). After the end of the third interaction, each subject completed a questionnaire assessing their engagement levels with various aspects of their experience with the robot (the Engagement questionnaire). Moreover, one week after their last interactions, the children completed exactly the same Knowledge and Habits questionnaire as they had completed before their first encounter with the system.

5.2.2 Subjects Three subjects participated in the case study: two female and one male, aged between 9 and 13 (Table 1). Each of them had type I diabetes and were recruited through a local patients' network association. All had three separate interaction episodes with the system, and two of the three had seen the Nao robot prior to the study (but had not interacted with the system). All relevant ethical guidelines were adhered to, including parental/guardian permission and data protection. The children had the opportunity to withdraw from the interactions and the study at any point if desired. 
Coninx et al., Towards Long-Term Social Child-Robot Interaction

\begin{tabular}{ccccc}
\hline Subject ID & Gender & Age & Familiar with robot & N(interactions) \\
\hline 1 & M & 9 & Yes & 3 \\
2 & F & 10 & No & 3 \\
3 & F & 13 & Yes & 3 \\
\hline
\end{tabular}

Table 1: Characteristics of the subjects who participated in the pilot study.

5.2.3 Experimental setup The multi-activity system used is as described above, with the robot theatre setup (section 4), involving the three activities and the conversation management interface. In addition to providing a rich and varied interaction, the three activities were used to teach different skills and pieces of knowledge to the child (Fig. 6). The creative dance activity involves physical activity, nonverbal interaction and short verbal exchanges, and addresses both the need for the children to exercise and the teaching of nutrition information. The collaborative sorting activity is mostly nonverbal and involves learning how to discriminate between different classes of food. The turn-taking quiz is mostly verbal interaction and involves learning various information about how to manage diabetes. The three activities also differ in the type of relationship they allow the child to experiment in with the robot, while maintaining the peer-to-peer interaction characteristic: The quiz is a competitive activity in which child and robot periodically switch roles (who asks and answers the questions), the sorting game is a collaborative activity in which the two participants attempt to solve the same sorting task together, while in the dance activity, the robot behaves more as a knowledgeable peer attempting to impart knowledge to the child.

The robot acted partly autonomously during the interaction. The control was shared between the system's autonomous components and the wizard's oversight, with the level of autonomy depending on the activity and context. Most of the robot's behaviours and reactions were controlled by the integrated system in an automated way, with the wizard's role mostly being to give general highlevel directives to control the user interaction session (when to start, stop, pause an activity, an interaction, etc.) and to make up for shortfalls in the current state of the art. The most notable tasks performed by the wizard were:

- Session control tasks: The human operator's task entailed deciding when interaction starts and ends, and overseeing the starting, stopping, pausing and resuming of activities, taking into account the information provided by the activities and the subject's state and desires, according to the guidelines described in Fig. 7;

- The conversation management verbal interaction system: This was implemented as a set of preset dialogue choices, selected by the operator;

- Evaluation of the subject's move during the Creative Dance activity: The general flow of the activity was completely automated, but the operator had to regularly give feedback on the quality of the postures and motions performed by the subject;

- Evaluation of the subject's answers during the Quiz activity: The activity processed mostly autonomously, but in the absence of reliable speech recognition, the operator was required to check which answer the subject had selected in response to a question;

- Physical safety oversight: Each activity directed robot motion in an autonomous way, but the operator could take over to address issues such as the robot falling, behaving dangerously or facing an inappropriate direction.

Due to of the modular design of our system and the rule-based nature of the wizard interventions, each of those specific cases where the interaction makes use of the wizard could be readily replaced by a fully autonomous system, should the appropriate sensory and cognitive components 
The robot paused the activity in the following conditions:

- The operator or the psychologist wanted to engage in off-activity talk $\mathrm{k}^{a}$;

- The child needed to temporarily leave the robot theatre.

The robot asked the child if they wanted to go on with the current activity and stopped the activity if asked to, in the following conditions:

- The child explicitly expressed wanting to switch activity or stop the current game;

- The child stopped playing with the robot or otherwise did not seem engaged in the activity;

- The child looked bored;

- The child had been playing the current activity for 10 minutes or more ${ }^{b}$.

The robot authoritatively stopped the activity in the following conditions:

- The child had been playing the current activity for 20 minutes or more;

- The time slot allocated to the child in the experimental schedule was about to be over;

- The current activity encountered a technical issue (this did not occur in the current study);

- The current activity had exhausted its content for the session (for example, all quiz questions had been asked: this did not occur in the current study).

\footnotetext{
${ }^{a}$ Off-activity talk was used when the operator perceived a drop of interest in the child, or when a suitable conversation topic arose from the current activity's content or was mentioned by the child.

${ }^{b}$ Since some activities include "critical sections" during which they can't be interrupted, the actual proposal to switch activity could occur slightly later than 10 minutes after the start of the activity.
}

Figure 7. Rules followed by the wizard operator to handle the switching/pausing/stopping of the activities in response to child behaviours and timing constraints.

be developed. An overview of how such an increased autonomy could be achieved is discussed in section 6.

5.2.4 Measurements Three types of measurement and data were applied and collected over the course of the proof-of-concept case study: questionnaires, structured interviews, and withininteraction data collection. Each of these provides a different perspective on the children's experiences and attitudes as they took part in the study, enabling an insight into the effectiveness of the multi-activity switching system. In addition to this, all interactions were video recorded for further qualitative analysis (a number of screenshots are shown below from these recordings).

Questionnaires: These were administered to assess knowledge levels (Appendix A1) and dietary/lifestyle habits (Appendix A2), and to derive feedback and impressions of children regarding the robot system and their interactions with it (Engagement questionnaire, Appendix A3). The knowledge and lifestyle questionnaires were based on published dietary guidance (European Food Information Council, 2009; Office of Disease Prevention and Health Promotion, 2013; World Health Organization, 2014), with a particular emphasis added on diabetes management. The engagement questionnaire was based on the ACL approach (Gough \& Heilbrun, 1980) and employed Likert-style question responses made more friendly for the children by using a progression of smiley/frowny faces instead of a numerical scale (where appropriate).

Structured Interviews: Over the course of the interaction sessions, a number of interviews with the children and their parents were conducted in order to discover their personal impressions about 


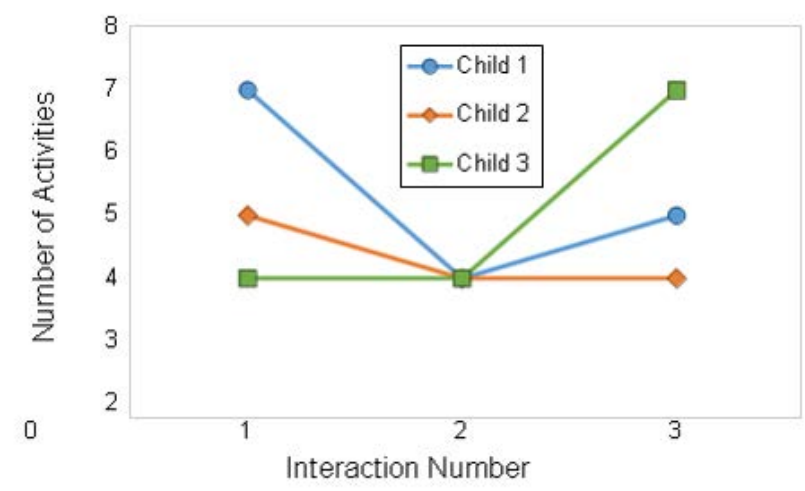

Figure 8. Number of different activities engaged in by each child in each session, taking into account activity switches and breaks in the interaction.

the system and its potential. In addition to this, a team of clinical stakeholders, who were involved in aspects of the activity contents to varying extents, were interviewed about their perspectives. This team was comprised of a psychologist, a paediatric diabetologist, a nutritionist, and the president of the diabetes patient association. See section 5.3.3 for the structure and outcome of these interviews.

Interaction Data: These were automatically logged by the robot system during the interactions. This included performance data, timing of child and robot behaviours, events arising in the interaction (such as interaction with the touchscreens) and the relative timings thereof. These data enable an objective characterisation of the child's behaviour within the interactions.

\subsection{Results}

5.3.1 Data collected during the interaction The planned session duration (for organization and scheduling purpose) was 50 minutes; actual sessions lasted 38 to 64 minutes, with an average duration of 48 minutes ( $S D: 9$ minutes) across the 9 sessions. All three children completed three full experimental sessions.

The child and robot engaged with between 4 and 7 different activities $(M: 4.89, S D: 1.27)$ during each session (Fig. 8). Different activities in this context mean that an activity was engaged in, left for a period of time, and then returned to later. For example, in session 2, Child 3 took a momentary break in the turn-taking quiz before resuming: For our statistics, this constitutes engagement in another activity. The variation of the number of activities engaged in over the three interaction session by Child 1 ( $M: 5.33, S D: 1.53)$, Child 2 ( $M: 4.33, S D: 0.58)$ and Child 3 ( $M: 5, S D: 1.73)$ provide an initial indication that a degree of personalisation was present, and that in the interactions, the children had the opportunity to express their activity preferences.

The content of the human-robot interaction sessions and the activity switching dynamics have been additionally studied for each child within the three interactions (Figs. 9, 10 and 11). The time series plots show that activity switching was widely used, and in all three interactions, each of the three activities was practised (though to differing extents).

FFor each child, the first interaction is relatively balanced between the three activities, as the child discovers them and understands having self-determination over which activity will be practiced (Figs. 9a, 10a and 11a). But apart for the second child, that behaviour changes in further interactions. Child 3 spends much time in the quiz activity (61\% of activity time in the second interaction and $50 \%$ in the third interaction) to the detriment of other activities (Fig. 11d). Conversely, Child 1 does not seem to enjoy the quiz activity much and spends only $14 \%$ of activity time practising it in the 


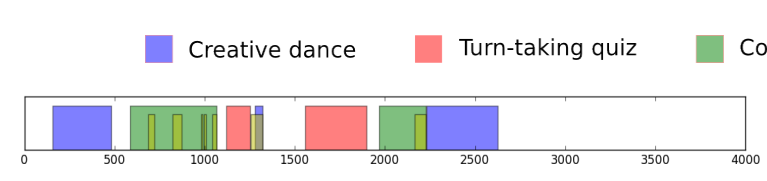

(a) Child 1, interaction 1

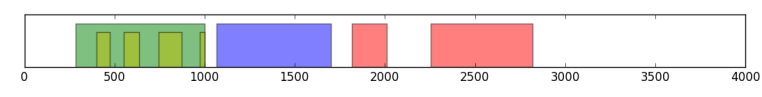

(b) Child 1, interaction 2

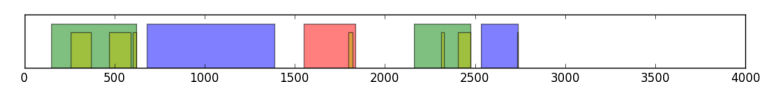

(c) Child 1, interaction 3
Collaborative sorting Activity paused

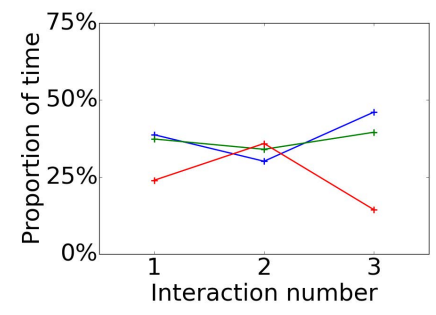

(d) Relative time spent in activities

Figure 9. Activities engaged in by Child 1 during the interactions. Figs. 9a, $9 \mathrm{~b}$ and $9 \mathrm{c}$ depict the sequence of activities during each of the three interactions. Time is given in seconds elapsed after the moment when the robot greets the child. Pauses were mostly triggered at the operator's request to engage in small talk or off-activity talk (i.e. informal verbal exchanges around topics that directly arise from the activity), and gaps between activities correspond to activity and session management talk (see section 4.2). Fig. 9d shows the evolution of the relative proportion of time spent in each of the three activities across the three interactions. The time spent with the activity paused is included in the computation, since most of this time is spent on off-activity talk.

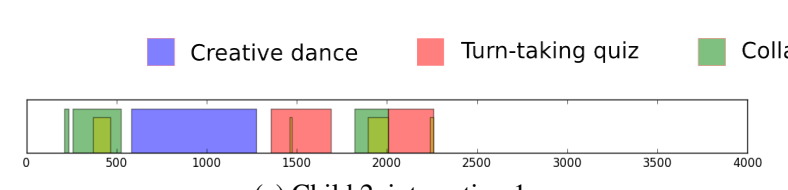

(a) Child 2, interaction 1

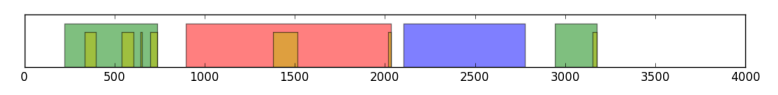

(b) Child 2, interaction 2

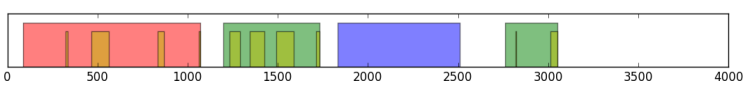

(c) Child 2, interaction 3

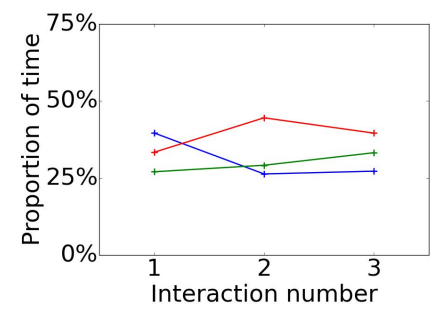

(d) Relative time spent in activities

Figure 10. Activities engaged in by Child 2 during the interactions (see fig. 9 caption for details). 


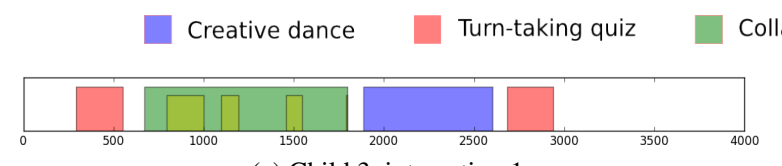

(a) Child 3, interaction 1

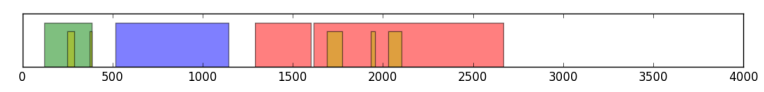

(b) Child 3, interaction 2

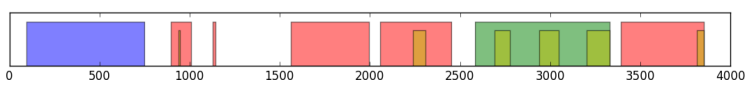

(c) Child 3, interaction 3
Collaborative sorting Activity paused

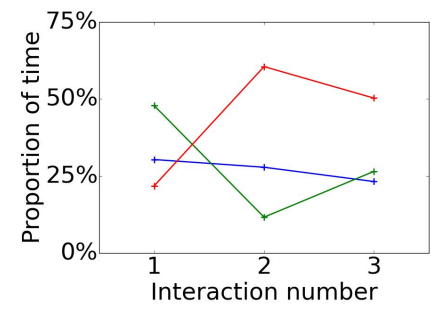

(d) Relative time spent in activities

Figure 11. Activities engaged in by Child 3 during the interactions (see fig. 9 caption for details).

last session, instead focusing on the dance and sorting activities (Fig. 9d). The maximal time spent within an activity is partially constrained by the switching rules employed by the wizard, but these figures nevertheless show that personal preferences were exercised by the children.

This personalisation of the interaction structure is the main point to emerge from these interaction statistics, as related to the central issue of motivation in long-term interaction and as raised above in section 2 .

5.3.2 Questionnaire data Qualitative measurements regarding the interaction and its perception by the children can be obtained by examining the differences in scores for the Knowledge and Habits questionnaires before and after the interactions along with processing the Engagement questionnaire. Given the narrow poll of participants involved in the experience described, the results obtained through the questionnaires administered to children have to be considered only as a preliminary case study, providing indicative data and exploring the potentialities of the system under analysis.

The scores of the Knowledge questionnaire show that all three children improved or remained stable in their total number of correct answers in the Knowledge questionnaire (Table 2). Two out of three children increased their knowledge on the topics covered by the three activities, especially in questions related to nutrition. The normalised learning gain results, which control for pre-test score given a limited scope for improvement (Meltzer, 2002), provide a similar perspective. These results provide an initial indication that the proposed system has a potential role to play in support of knowledge gain.

\begin{tabular}{lccc}
\hline & Pre-Knowledge test & Post-Knowledge test & Learning gain $(g)$ \\
\hline Child 1 & 8 & 8 & 0.0 \\
Child 2 & 7 & 8 & 0.143 \\
Child 3 & 9 & 10 & 0.2 \\
\hline
\end{tabular}

Table 2: Results of the pre- and post-test Knowledge scores, with resulting learning gain. The score is computed by counting 1 point per correct answer for each of the 14 items (see questionnaire in Appendix A1) for a maximum score of 14. The learning gain $g$ varies between 0 and 1 and is computed as described in Meltzer (2002). 
Coninx et al., Towards Long-Term Social Child-Robot Interaction

\begin{tabular}{lcccc}
\hline & Turn-Taking quiz & Creative dance & Collaborative sorting & Average \\
\hline Child 1 & 4 & 5 & 4 & 4.33 \\
Child 2 & 4 & 4 & 5 & 4.33 \\
Child 3 & 4 & 5 & 5 & 4.67 \\
\hline
\end{tabular}

Table 3: Ratings of the individual activities by the children on a 5-point Likert scale as part of the Engagement questionnaire (items 7, 8 and 9, see questionnaire in Appendix A3.) The individual rating of each activity by each child is reported, as well as the per-child average over all activities.

The Habits questionnaire score did not show significant differences between before and after the intervention period. This lack of difference could be a consequence of known issues with the administering of questionnaires to children, such as ceiling effects or desires to please the experimenter (Richman, Kiesler, Weisband, \& Drasgow, 1999). This could also indicate that although three hourlong interactions with the robot over a period of one month may teach the children some knowledge, these are not of sufficient length to elicit changes in habitual behaviour outside of the interaction setting. However, this non-trivial issue encompasses a wider range of contributing factors (e.g. home and school environments, etc.), which constitutes an important area of future investigation.

From a qualitative point of view, a positive perception of the robot emerges. The Engagement questionnaire shows that all of the children appreciated the robotic character and the activities proposed in the robot theatre: on the basis of a 5 point incremental Likert scale (going from $1=$ "I did not like it at all" to $5=$ "I liked it very much"), the Nao robot scored 4.3, and the mean score for all the activities is 4.4 (see Table 3). Moreover, all three children expressed desire to play with the robot again in the future.

The detailed comments and feedback from the children place the robot in a humanized dimension and, in particular, in a relational sphere. For example, in the multi-adjective choice section of the Engagement questionnaire, children chose words such as "smart" and "lovely" (i.e. adjectives normally used to describe a human being) or "kind" and "fun" (normally linked to a relational context) to describe Nao. None of the negative adjective (e.g. "boring", "mechanical", "fake", etc.) were chosen. To support this idea, all three children identified the robot's emotional state as happy, and two of the three attributed to it the ability to recognize their emotions. On closer inspection, these two specific children where those who already had the opportunity to interact with the robot in the past, while for the third child this was the first contact with Nao. This result may suggest that an experience of long-term interaction allows the creation of a relation that assumes an emotional tone for a child.

5.3.3 Stakeholder interviews Structured interviews were conducted with all stakeholders involved in the process of supporting the children in their learning about diabetes self-management: the children themselves, their parents, medical professionals at the hospital, and patient support networks. These interviews were conducted by a member of the technical research team, under the direction of the research psychologist.

Child Interviews: The interviews with the children covered a range of topics, expressed in an appropriate way for the age range and for the individuals. The main emphases were firstly regarding their interactions with the robot (what they liked/disliked about the robot itself, their interaction with it, the activities, their feelings about the questions the robot asked them ${ }^{3}$, etc). Secondly, they were

\footnotetext{
${ }^{3}$ These questions concerned the small talk and off-activity talk introduced by the technical wizard, guided by the psychologist.
} 


\begin{tabular}{|c|c|c|c|}
\hline & Children & Parents & Both \\
\hline 1. & Playful aspect & Attractiveness for all children & $\begin{array}{l}\text { Share feelings and emotions } \\
\text { about diabetes }\end{array}$ \\
\hline 2. & Learning tool for all children & $\begin{array}{l}\text { Openness towards the robot } \\
\text { due to perception of it as a } \\
\text { companion }\end{array}$ & $\begin{array}{l}\text { Learn something new and } \\
\text { useful }\end{array}$ \\
\hline 3. & $\begin{array}{l}\text { Distraction from pain related } \\
\text { to disease }\end{array}$ & $\begin{array}{l}\text { Humanized attributes of the } \\
\text { robot }\end{array}$ & Robot in multiple contexts \\
\hline 4. & $\begin{array}{l}\text { Companionship during hospi- } \\
\text { talization }\end{array}$ & & \\
\hline 5. & $\begin{array}{l}\text { Self efficacy from teaching } \\
\text { the robot about diabetes }\end{array}$ & & \\
\hline
\end{tabular}

Table 4: Key concepts emerging from the interviews with the children involved in the study and their parents.

asked for their opinions on how the system could be used in a wider context (would they like to see the robot again, and if so, what would they like to do, where and how could the robot be used in other applications, etc.).

From the interviews with the children, we obtained positive feedback about their interactions with the robot and about the role of the robot in the different activities proposed by the system (see Table 4). Moreover, they did not feel uncomfortable answering personal questions requested by the robot, even questions about diabetes: They thought the robot wanted to know them better and learn something about diabetes, so they were well disposed to speak about such a delicate subject. (Child 3: "When it [Nao] told me I can ask you some questions about diabetes, I was happy, because I knew well the answer"). Children felt empowered through their participation in the project, because they thought they were contributing to the robot's development (Child 1: "I liked questions about diabetes, because it was a chance for Nao to learn something from me.") One of the most interesting pieces of feedback was regarding the possible support that the robot could provide to children admitted to the hospital for any medical conditions, cheering their days spent in an unfamiliar environment. (Child 1: "It [Nao] could play with them [the hospitalized children] if they are alone. Or make them forget the bad things that they have gone through because of their illness"). While there are some issues with interviewing children (Richman et al., 1999), these opinions are consistent with the findings of other experiments using alternative interrogation methodologies (Baroni et al., 2014).

Parent Interviews: The interviews with the parents were conducted while their children were interacting with the system; the children were therefore not present. Each of these interviews were conducted following the same structure: firstly, impressions of robots in general, and then more specifically, the Nao robot; secondly, their motivations in allowing their children to take part in the study, including their expectations; and finally, regarding their observations of their child interacting with the system, including any conversations they had had with their children on the subject. For Child 1, both parents were interviewed together. For Child 2, only the mother took part by, providing general impressions. For Child 3, the parents were interviewed separately.

All parents thought that the interaction with the robot through such a gaming platform could be useful to teach knowledge about nutrition and diabetes, but they also stated that the interaction could be based on other topics that even non-diabetic children could benefit from, for example, in a 
more generic educational context, where such interaction could improve the teaching of all subjects. (Mother of Child 3: "It could be useful to have it at school... Because maybe it's easier to let them learn the things said by a cute little robot rather than have them taught by the teacher").

Some other relevant observations were made on parents' opinions and reactions regarding their child's involvement in the study (Table 4). Three aspects were generally observed: the potential of the integrated system in educational matters, the description of Nao with humanlike characteristics (father of Child 1: "For me it is important that the child expresses its sensitivity to the topic [diabetes] to an object that is not human"), and verification of their child's knowledge and habits outside their house. All parents agreed that the activities with the robot could be proposed in schools (amongst other structures, like hospitals, waiting rooms, etc.) in order to educate diabetic children on nutrition and physical activities, but also to increase awareness of diabetes for non-affected persons. Moreover, most of the parents concur with the medical staff in seeing a high potential for the system developed to teach other subjects and topics, not necessarily linked to diabetes or health (father of Child 1: "...in any context where there are children, like hospitals or schools, educational trips or other experiences that relate to any child. For me, the potential is very high").

Medical and Patient Network Staff: Structured interviews were conducted with a psychologist, a paediatric diabetologist, a nutritionist, and the president of the diabetes patient association. These healthcare professionals were directly involved in the treatment and support of children with diabetes in the hospital and outside of it. The interviews covered four topics: first, the role and relevance of the activities and their design to the application; secondly, the potential of the system as a whole for education and support of children with diabetes; thirdly, what would be required to improve the relationship between the robot and the children; and finally, whether there were other suitable and appropriate domains of application for the robot system (or a variation of it).

In general, all four expressed positive opinions of the system and its potential as an educational support tool (nutritionist: "Nao could be an intermediary in the work of education..."), not just related to diabetes, but also to other domains (patients association: "I believe that the outcomes of this project should be used not only to support the medical educational process, but also to raise awareness about prevention of chronic diseases (...) like, for example, in schools").

More specifically, a number of benefits of such a robot-supported learning approach were raised. One of the main ones of these were regarding the support of motivation (e.g. diabetologist: "The contribution of the Nao could be in this perspective: support to learning and motivation") and in providing an environment in which the children are at ease (psychologist: "When children enter in the (...) robot system (...) setting they arrive in a relaxed and gaming dimension, that's familiar to them. Therefore, children meet a condition that activates them positively (...), where there is no judgement"). Intriguingly, there was also a potential role envisaged for the robot as an intermediary between the child and the medical staff (nutritionist: “... supporting the work of doctors, to pass messages that can be useful for these children to care for them properly with the least difficulty possible").

\section{Discussion}

\subsection{Case study result}

The main goal of the experimental evaluation of our integrated system was to assess the potential of using a robotic system integrating multiple activities in a coherent behavioural framework to support a richer and more personalized user experience and to examine the real and potential consequences of that improvement in the domain of supporting children with diabetes in their learning how to self-manage their condition. Our results show that the activity switching mechanism is actively used by the children to customize their interaction with the robot, demonstrating the interactions are 
personalised to the preferences of the individual child. The qualitative reports support this effect by highlighting that the children generally have a positive view of the interaction with the robot. Thus, while further intervention evaluation is required to quantitatively demonstrate the effect, our claim that activity switching within individual interactions can support long-term interaction (and then potentially knowledge gain and habits change) is provided with support from this case study. Personalisation of interactions can lead to more sustained motivation and learning (Dweck, 1986), a point supported by the views of the interviewed diabetologist.

The low number of subjects in our case study limits the statistical conclusions that can be drawn from those results, but that limitation also allowed a focus on a finer analysis of each session, which is relevant for a system that puts the emphasis on personalising the interaction. The first conclusion is that children exhibit different preferences and adjust the time spent in various activities accordingly: for example, Child 1 seems to dislike quiz (relative to other activities, Fig. 9d) while Child 3 prefers it to other activities (Fig. 11d). However, we also see that even when the subject shows no significant preferences and divides his time equally between all three activities, as appears to be the case for Child 2 (Fig. 10d), the activity switching is used often (Fig. 8), allowing the child to split the hourlong session into shorter episodes (the mean time spent in an activity between switching to another activity or terminating the session is 9 minutes), which can be instrumental to keeping the child engaged with the interaction during an extended period of time.

Although our user study is relatively limited in terms of length of time of interaction, this contrasts with reports of other experiments using less varied interactions where, despite being globally happy with the interaction, users often showed or reported signs of boredom and became less engaged in the system as the novelty effect wore off (Ros, Baroni, \& Demiris, 2014), or they gradually chose to spend less and less time with the robot when presented with a single activity with no form of individual adaptation (Janssen et al., 2011), sometimes only after a few minutes. Conversely, children using our system remained interested and motivated throughout three 50-minute sessions, spending time playing and speaking with the robot and expecting a high level of social intelligence from it. While the time periods covered in the present case study may not have been sufficient to objectively state that boredom was permanently avoided in the children who took part, a comparison to these prior works nevertheless demonstrates an increase in sustained attention. Over additionally extended periods of time, the effect of boredom and disinterest may yet become apparent when using our proposed approach, however, we suggest that this may then involve mechanisms of child motivation for learning that extend beyond the capacities of an individual system such as ours, encompassing children's perception of learning goals and structures (e.g. Ames, 1992), and more general interest in the topic (e.g. Schiefele, 1991).

6.2 The multi-activity integrated system: A step closer to rich, autonomous child-robot interaction

In this paper, we put forward a multi-activity system that can be used to provide richer and more varied interactions for cHRI. The three activities that were used within the framework of our system were chosen and designed to constitute complementary elements, used to fulfil a common goal through different means. All were conducted in the general context of the problem domain of our study (supporting hospitalized diabetic children), but they explore different ways the child and robot can interact: mostly verbal (in the quiz), mostly nonverbal (in the collaborative sorting activity) or a mixture of both (in the creative dance activity); the activities can be competitive (the quiz) or collaborative (collaborative sorting); and the child and robot's role in the relationship can be identical (two partners playing the same game together, as in the collaborative sorting activity) or different (one agent teaching the other, as in the creative dance activity). We posit that this diversity can help adapt the interaction to each child's preferred relationship style and cognitive preferences.

The system described and used in this paper only provides three different types of interactions 
linked through an activity switching and behaviour control mechanism. Although this system is fully functional and has been shown to be usable in a real healthcare application, we must acknowledge that it is only a fairly crude approximation of the elaborate social behaviours that humans routinely show in (and expect from) dyadic interactions. This may cause issues in some cases, since subjects may over-estimate the social abilities of the robot they are engaged with. Whereas human users interacting with a simple system able to engage only in a single type of activity are able to relatively quickly understand its limitations, the subjects using our multi-activity system will no doubt expect it to have a more flexible and autonomous behaviour than it is currently able to show. The system we propose is only a first step towards building more flexible, personalised and context-aware humanrobot interaction, and we hope the challenges it raises can provide a wider context for the work and set up the discussion of future research avenues.

Concerning the autonomous behaviour of the robot, we wish to highlight that although some important features of our integrated system still require supervision from an operator to be functional (see section 5.2.3), the system is not built around components that must be manually controlled: The modular, layered architecture paves the way for replacing the GUI and human supervision by components providing the same functionalities in an autonomous way when they are developed, in accordance with the recommendations on WoZ studies (Kelley, 1984; Riek, 2012). The activities already provide differing competencies in this regard. The collaborative sorting activity (Baxter et al., 2012) is already mostly autonomous and requires very little supervision. The creative dance activity only requires feedback from the operator on the child's performance, and this requirement is being phased out with the development of new methods based on motion sensing that can perform this evaluation in an automatic way. The quiz activity still requires some direct control from the operator, although improvements in the speech recognition components should also alleviate this requirement in the future.

At a higher level, replacing - or complementing - the wizarded activity selection GUI with an autonomous selection module able to automatically perform the activity switching and selection poses no additional technical integration difficulties. The wizard operated under a defined set of rules to manage the switching process (a process informed by the clinical psychologist, see section 5.2.3), which lent itself to automation. Indeed, the manner in which the rules were formulated means that the wizard was encouraged to take a robot-centric (as opposed to a higher-level goalcentric) perspective, meaning that the wizard operating rules can be mapped directly onto the actions currently directly available to the robot.

Nevertheless, the development of such an autonomous component poses still unresolved issues, due to the complex nature of the activity selection and switching task. One important strength of the multi-activity system is that it allows children to enjoy a more varied and interesting interaction with the robot; a goal of the autonomous activity switching system should therefore be to keep the child interested and attentive during the whole interaction by switching activities to prevent boredom and repetitiveness. However, the general goal of the child-robot interaction in our application domain, knowledge gain, should also be taken into account for selection, since all activities have different informational content, and those two objectives may not be in agreement. Finally, the activity selection is constrained by logistic issues (limited interaction time, discharging robot battery, etc.) and the potentially limited intrinsic content of activities (for example a quiz game can conceivably run out of relevant questions). The way these different factors have to be prioritized, combined and taken into account in an autonomous activity selection system has to be clarified before such a system can be built. One potential solution to this problem, assuming that the state space can be adequately represented by the available sensory competencies (for example in determining the engagement of the interacting child), lies in the application of machine learning techniques to what may be characterised as a multiple-goal optimisation problem. Experiments following the structure of the case 
study we present here can provide the data collection opportunities necessary to provide training data for such methods.

\subsection{Balancing desiderata and constraints}

The design of the case study is the result of a compromise between several mutually antagonistic factors and objectives. Our desire to study long-term interaction with children in an ecologically valid environment (i.e. a hospital rather than a computer science laboratory) directly challenges the requirements of HRI research, which needs extensive data collection during well-defined interactions in a controlled experimental environment that can most easily be achieved in lab conditions. Besides, unlike some previous work on long term child-robot interaction that focused mostly on the interaction between the robotic agent and a group of children (e.g. a school class; Tanaka et al., 2006) taken as a whole, we specifically studied personal interaction and adaptation based on the individual preferences of the child. Social robotics is still an emerging field, and the contexts that make it possible to engineer a credible situation where child and robot interact on a long-term basis with appropriate instrumentation and data recording are still few. In this regard, the use case of companions for diabetic children in a hospital setting was a unique opportunity, since it allowed us to present the robot interaction as both a research program and a therapeutic tool (to support existing structures), and made it possible to run the experiments in a semi-controlled environment that can be adapted to experimental purpose through the robot theatre system. The drawback of the present use case is the small number of subjects in our case study, which directly reflects the modest size of the target patient population and the restrictions of working in a hospital setting where scientific goals must naturally always give way to patient welfare and medical necessities.

It is important to consider how those strengths and limitations of the experimental setup interact with the strengths and limitations of the technical system that were discussed in section 6.2 and modulate the significance of the experimental results. Although the present study clearly shows the potential of a personalised interaction with multiple frameworks of activity, this conclusion is only based on at most three hours of interaction-split over three sessions-for each subject. It seems likely that the interest from the children would eventually have waned had more experimental sessions been conducted, but we remain uninformed about when that would have happened. On the other hand, even if we can not affirm that a varied, personalized interaction is sufficient to maintain motivation for an extended period of time, there is little doubt that the level of personalization we propose is necessary for any real long-term social robotics work. Our research therefore explores technologies that are required for long-term interaction, even though we do not achieve true longterm interactions at present, as is the case with other currently existing research efforts.

We have used questionnaires, interviews and data recorded by the system to evaluate our case study and show that our system can successfully be used in ecological conditions, and that the multiactivity feature actively contributes to this success. It is to be noted that some effects, such as the individual preferences of each child for specific activities, can only be detected through analysis of the technical data collected by the system (Figs. 9, 10 and 11) and not in the self-report questionnaire scores which exhibit a strong ceiling effect (Table 3). There are however still open questions regarding how to evaluate the behaviour of such a multi-activity system as an HRI framework. Even in smaller robotic systems that focus on one framework of engagement, finding the specific tools, measurements and metrics that are best suited to study the interaction can be challenging. Since the multiple activities we use define independent frameworks that constrain the nature and the goal of the interaction, a new problem is put forward: how to study the relationship across several different successive forms of interaction, during which the child and the robot engage in different activities and potentially different types of social behaviour? Even evaluating the same social dimension across multiple activities may require a modification of the methodologies applied. For example, 
in a scenario where the robot and the child are interacting with each other with no other object present, monitoring the child's gaze direction can provide a metric of the attention paid to the robot (e.g. Sidner et al., 2005; Sidner, 2012). But in another activity where the robot and the child are jointly using another device (such as a touchscreen), the child must divide attention between the robot and the device (Baxter, Kennedy, Vollmer, de Greeff, \& Belpaeme, 2014). That makes using that measurement, and more pertinently its implications, more complex. Intrinsic data directly provided by the activity (such as game scores, timing and correctness of user responses, etc.) can also be very heterogeneous across activities, which makes it difficult to treat as a whole body of data about the interaction, even if the informational content is about similar characteristics of the interaction. We therefore advocate not only a pluralistic approach to robot behavioural and interactional competences (as evident in our multi-activity framework), but also a corresponding approach to cHRI evaluation that integrates evidence from a wide range of potentially codependent metrics from instantaneous to long-term behavioural changes.

The fact that all stakeholders (parents, medical staff, educators, etc.) expressed an interest for the robotic system and saw a potential for it to be used in a variety of contexts and for multiple education and support applications, shows that the integrated system we designed has potential both as a cHRI research tool and as a real educational and therapeutic addition to existing structures and procedures. Although the specific setup we used is an initial system that requires further refinement, the principles we developed over the course of our efforts, and described in this paper, provide a foundation for exciting new developments. As cHRI moves further out of the lab and attracts interest as a tool for children support and education, more incentive will be provided to run cHRI systems "in the wild" on a regular, long-term basis. This will help overcome some of the barriers to the study of long-term child-robot interaction (such as the issues described in section 3.1) and allow for data collection and hypotheses testing to further improve the social behaviour of those robots.

\section{Conclusion}

The work presented in this paper attempts to extend the scope of cHRI research by overcoming the limitations of the approaches focused on one activity only. The multi-activity approach we propose still has limitations, and its use poses specific technical and scientific challenges, some of which were circumvented in the present study through the use of WoZ methods. However, we have shown that it allows us to study multiple, discontinuous, hour-long child-robot interactions during which the child shows a high level of engagement and educational or therapeutic goals can be pursued.

Our approach has child and robot meet in the open environment of the robot theatre, where they can talk together and jointly decide how they want to spend time together. Although our simulation of a free interaction still requires refinement (with only three activities and off-activity talk), the children were unanimous in their positive reaction to this approach, as indeed were their parents and the medical professionals involved in their care. The preliminary results from the presented hospital-based case study demonstrate that putting the interaction in this open framework could lead to richer, deeper and more human-like social interactions by taking into account personal preferences regarding interaction and activity type. While we are still far from being able to account for the infinitely complex social interactions children engage in during interaction with each other and with adults, our proposed multi-activity framework therefore constitutes a modest but significant step in that general direction.

Feedback from the range of stakeholders involved in the case study show that they deem the abilities shown by our multi-activity system to be good enough to be useful outside of the lab for real educational and therapeutic use. Our approach therefore seems especially promising for setting up long-term field social robotics experiments to both study the evolution and dynamics of a child-robot relationship over several weeks or months of interaction. While there is further experimentation 
yet to be conducted to verify the efficacy of such a multi-activity child-robot interaction context on longer-term knowledge gain and behaviour change, we have provided the groundwork for such empirical investigation in this paper.

\section{Acknowledgements}

This work is supported by the EU Integrated Project ALIZ-E (FP7-ICT-248116). The authors wish to thank the SOStegno 70 association for diabetic children and the team of the Center for Pediatric and Adolescent Endocrinology of Ospedale San Raffaele for their constant support of this research.

\section{References}

Ames, C. (1992). Classrooms: Goals, structures, and student motivation. Journal of Educational Psychology, 84(3), 261-271. doi:10.1037/0022-0663.84.3.261

Baillie, J.-C. (2005). URBI: Towards a universal robotic low-level programming language. In Proceedings of the 2005 IEEE/RSJ International Conference on Intelligent Robots and Systems (IROS 2005) (p. 820825). doi:10.1109/IROS.2005.1545467

Baillie, J.-C., Demaille, A., Hocquet, Q., Nottale, M., \& Tardieu, S. (2008). The Urbi universal platform for robotics. In Proceedings of the First International Workshop on Standards and Common Platform for Robotics (SIMPAR 2008) (pp. 580-591). Venice, Italy.

Baroni, I., Nalin, M., Baxter, P., Pozzi, C., Oleari, E., Sanna, A., \& Belpaeme, T. (2014). What a robotic companion could do for a diabetic child. In Proceedings the 23rd IEEE International Symposium on Robot and Human Interactive Communication (RoMan '14) (pp. 936-941). Edinburgh, U.K.: IEEE Press. doi:10.1109/ROMAN.2014.6926373

Bartneck, C. (2003). Interacting with an embodied emotional character. In Proceedings of the 2003 International Conference on Designing Pleasurable Products and Interfaces (DPPI '03) (pp. 55-60). Pittsburgh, USA: ACM Press. doi:10.1145/782896.782911

Baxter, P., Baroni, I., Nalin, M., Sanna, A., \& Belpaeme, T. (2013). Touchscreens as mediators for social human-robot interactions: A focus group evaluation involving diabetic children. In Proceedings of the CmIS workshop at ITS '13. St Andrews, U.K.

Baxter, P., deGreeff, J., \& Belpaeme, T. (2013). Cognitive architecture for human-robot interaction: Towards behavioural alignment. Biologically Inspired Cognitive Architectures, 6, 30-39. doi: 10.1016/j.bica.2013.07.002

Baxter, P., Kennedy, J., Vollmer, A.-L., de Greeff, J., \& Belpaeme, T. (2014). Tracking gaze over time in hri as a proxy for engagement and attribution of social agency. In Proceedings of the 2014 ACM/IEEE International Conference on Human-Robot Interaction (HRI'14) (pp. 126-127). Bielefeld, Germany: ACM Press. doi:10.1145/2559636.2559829

Baxter, P., Wood, R., \& Belpaeme, T. (2012). A touchscreen-based "sandtray" to facilitate, mediate and contextualise human-robot social interaction. In Proceedings of the 7th ACM/IEEE International Conference on Human-Robot interaction (HRI) (pp. 105-106). Boston, MA, U.S.A.: ACM/IEEE Press.

Beck, A., Canamero, L., \& Bard, K. (2010). Towards an affect space for robots to display emotional body language. In Proceedings of the 19th IEEE International Symposium in Robot and Human Interactive Communication (RoMan 2010). doi:10.1109/ROMAN.2010.5598649

Beck, A., Hiolle, A., \& Canamero, L. (2013). Using Perlin noise to generate emotional expressions in a robot. In Proceedings of the 35th Annual Meeting of the Cognitive Science Society (CogSci 2013) (pp. 1845-1850). Berlin, Germany.

Belpaeme, T., Baxter, P., Greeff, J. D., Kennedy, J., Looije, R., Neerincx, M., .. Coti, M. (2013). Child-robot interaction: Perspectives and challenges. In Proceedings of the 5th International Conference on Social Robotics (ICSR 2013) (pp. 452-459). Bristol, U.K.: Springer.

Belpaeme, T., Baxter, P., Read, R., Wood, R., Cuay, H., Kiefer, B., .. Humbert, R. (2012). Multimodal child-robot interaction: Building social bonds. Journal of Human-Robot Interaction, 1(2), 33-53. doi: 10.5898/JHRI.1.2.Belpaeme 
Beran, T. N., Ramirez-Serrano, A., Kuzyk, R., Fior, M., \& Nugent, S. (2011). Understanding how children understand robots : Perceived animism in child robot interaction. Journal of Human Computer Studies, 69(7-8), 539-550. doi:10.1016/j.ijhcs.2011.04.003

Billard, A. (2002). Play, dreams and imitation in robota. In K. Dautenhahn, A. Bond, L. Caamero, \& B. Edmonds (Eds.), Socially intelligent agents (Vol. 3, pp. 165-172). Springer. doi:10.1007/0-306-473739_20

Blanson Henkemans, O. A., Bierman, B. P. B., Janssen, J., Neerincx, M. A., Looije, R., van der Bosch, H., \& van der Giessen, J. A. M. (2013). Using a robot to personalise health education for children with diabetes type 1: A pilot study. Patient education and counseling, 92(2), 174-81. doi:10.1016/j.pec.2013.04.012

Blanson-Henkemans, O., Hoondert, V., Schrama-Groot, F., Looije, R., Alpay, L., \& Neerincx, M. (2012). "I just have diabetes": Children's need for diabetes self-management support and how a social robot can accommodate their needs. Patient Intelligence, 4, 51-61. doi:10.2147/PI.S30847

Christophel, D. M. (1990). The relationships among teacher immediacy behaviors, student motivation, and learning. Communication Education, 39(4), 323-340. doi:10.1080/03634529009378813

Cosi, P., Paci, G., Sommavilla, G., Tesser, F., Nalin, M., \& Baroni, I. (2012). An Italian event-based ASR-TTS system for the Nao robot. In Proceedings of the 7th Conference of the Italian Association of Speech Sciences (pp. 177-198). Rome, Italy.

Csala, E., Németh, G., \& Zainkó, C. (2012). Application of the NAO humanoid robot in the treatment of marrow-transplanted children. In Proceedings of the 3rd IEEE International Conference on Cognitive Infocommunications (pp. 655-659). Kosice, Slovakia.

Dautenhahn, K. (2004). Robots we like to live with?! A developmental perspective on a personalized, lifelong robot companion. In Proceedings the 13th IEEE International Symposium on Robot and Human Interactive Communication (RoMan'14) (pp. 17-22). Kurashiki, Japan: IEEE Press.

Dautenhahn, K. (2007). Socially intelligent robots: Dimensions of human-robot interaction. Philosophical Transactions of the Royal Society of London. Series B, Biological Sciences, 362, 679-704. doi: 10.1098/rstb.2006.2004

Draper, T. W., \& Clayton, W. W. (1992). Using a personal robot to teach young children. The Journal of Genetic Psychology, 153(3), 269-273.

Dweck, C. S. (1986). Motivational processes affecting learning. American Psychologist, 41(10), 1040-1048. doi:10.1037/0003-066X.41.10.1040

European Food Information Council. (2009, October). Food-based dietary guidelines in Europe. Website. Retrieved from http://www.eufic.org/article/en/ expid/food-based-dietary-guidelines-in-europe

Fine, A. (2010). Handbook on animal-assisted therapy (3rd ed.). San Diego, CA, USA: Academic Press.

Gough, H. G., \& Heilbrun, A. B. (1980). The adjective check list manual: ACL. Palo Alto, CA, USA: Consulting Psychologists Press, Inc.

Hiolle, A., Lewis, M., \& Cañamero, L. (2014). Arousal regulation and affective adaptation to human responsiveness by a robot that explores and learns a novel environment. Frontiers in Neurorobotics, 8, 17. doi: 10.3389/fnbot.2014.00017

Hyun, E., \& Son, S. (2010). Relationships between user experiences and children's perceptions of the education robot. In Proceedings of 5th ACM/IEEE International Conference on Human-Robot Interaction - HRI'10 (pp. 199-200). Osaka, Japan: ACM/IEEE Press.

Janssen, J. B., van der Wal, C. C., Neerincx, M. A., \& Looije, R. (2011). Motivating children to learn arithmetic with an adaptive robot game. In Proceedings of the 3rd International Conference on Social Robotics (pp. 153-162). Berlin, Heidelberg: Springer-Verlag. doi:10.1007/978-3-642-25504-5_16

Kanda, T., Hirano, T., \& Eaton, D. (2004). Interactive robots as social partners and peer tutors for children: A field trial. Human-Computer Interaction, 19, 61-84.

Kanda, T., Sato, R., Saiwaki, N., \& Ishiguro, H. (2007). A two-month field trial in an elementary school for long-term human-robot interaction. IEEE Transactions on Robotics, 23(5), 962-971. doi: 10.1109/TRO.2007.904904

Keller, J. (1987). Strategies for stimulating the motivation to learn. Performance and Instruction, 1-7. doi: $10.1002 /$ pfi.4160260802 
Kelley, J. F. (1984). An iterative design methodology for user-friendly natural language office information applications. ACM Transactions on Information Systems, 2(1), 26-41. doi:10.1145/357417.357420

Kennedy, J., Baxter, P., \& Belpaeme, T. (2013). Constraining content in mediated unstructured social interactions: Studies in the wild. In Proceedings of the 5th International Workshop on Affective Interaction in Natural Environments at ACII 2013. IEEE Press.

Kennedy, J., Baxter, P., \& Belpaeme, T. (2014). Children comply with a robot's indirect requests. In Proceedings of the 2014 ACM/IEEE International Conference on Human-Robot Interaction - HRI'14 (pp. 198-199). Bielefeld, Germany: ACM Press. doi:10.1145/2559636.2559820

Kennedy, J., Baxter, P., \& Belpaeme, T. (2015). Comparing robot embodiments in a guided discovery learning interaction with children. International Journal of Social Robotics, 7(2), 293-308. doi:10.1007/s12369014-0277-4

Kidd, C. D., \& Breazeal, C. (2008). Robots at home: Understanding long-term human-robot interaction. In Proceedings of the 21st IEEE/RSJ International Conference on Intelligent Robots and Systems (IROS 2008) (pp. 22-26). Nice, France: IEEE Press.

Komatsu, T., \& Abe, Y. (2008). Comparing an on-screen agent with a robotic agent in non-face-to-face interactions. In H. Prendinger, J. Lester, \& M. Ishizuka (Eds.), Intelligent virtual agents (lecture notes in computer science 5208) (Vol. 5208, pp. 498-504). Berlin, Heidelberg: Springer Berlin Heidelberg. doi:10.1007/978-3-540-85483-8

Kose-Bagci, H., Ferrari, E., Dautenhahn, K., Syrdal, D. S., \& Nehaniv, C. L. (2009). Effects of embodiment and gestures on social interaction in drumming games with a humanoid robot. Advanced Robotics, 23(14), 1951-1996. doi:10.1163/016918609X12518783330360

Kozima, H., \& Nakagawa, C. (2006). Social robots for children: Practice in communication-care. In Proceedings of the 9th IEEE International Workshop on Advanced Motion Control (pp. 768-773). Istanbul, Turkey: IEEE Press.

Kruijff-Korbayova, I., Cuayahuitl, H., Kiefer, B., Schroder, M., Cosi, P., Paci, G., ... Verhelst, W. (2012). Spoken language processing in a conversational system for child-robot interaction. In Proceedings of the Workshop on Child-Computer Interaction (WOCCI). Portland, USA.

Kruijff-Korbayova, I., Kiefer, B., Baroni, I., \& Zelati, M. C. (2013, December). Making human-robot quiz dialogue more conversational by adding non-quiz talk. In The 17th Workshop on the Semantics and Pragmatics of Dialogue (DialDam) (p. Poster). Amsterdam, The Netherlands.

Kruijff-Korbayova, I., Oleari, E., Baroni, I., Kiefer, B., Zelati, M. C., Pozzi, C., \& Sanna, A. (2014, August). Effects of off-activity talk in human-robot interaction with diabetic children. In Proceedings of the 23rd IEEE International Symposium on Robot and Human Interactive Communication (RoMan 2014) (pp. 649-654). IEEE Press. doi:10.1109/ROMAN.2014.6926326

Lee, K. M., Jung, Y., Kim, J., \& Kim, S. R. (2006). Are physically embodied social agents better than disembodied social agents? The effects of physical embodiment, tactile interaction, and people's loneliness in humanrobot interaction. International Journal of Human-Computer Studies, 64(10), 962-973. doi: 10.1016/j.ijhcs.2006.05.002

Leite, I., Castellano, G., Pereira, A., Martinho, C., \& Paiva, A. (2012). Modelling empathic behaviour in a robotic game companion for children: An ethnographic study in real-world settings. In Proceedings of the 7th ACM/IEEE International Conference on Human-Robot Interaction - HRI'12 (pp. 367-374). Boston, MA, U.S.A.: ACM Press.

Leite, I., Martinho, C., \& Paiva, A. (2013). Social robots for long-term interaction: A survey. International Journal of Social Robotics, 5(2), 291-308. doi:10.1007/s12369-013-0178-y

Leyzberg, D., Spaulding, S., Toneva, M., \& Scassellati, B. (2012). The physical presence of a robot tutor increases cognitive learning gains. In Proceedings of the 34th Annual Conference of the Cognitive Science Society (pp. 1882-1887). Sapporo, Japan.

Looije, R., van der Zalm, A., Neerincx, M. a., \& Beun, R.-J. (2012). Help, I need some body: The effect of embodiment on playful learning. In Proceedings of the 21st IEEE International Symposium on Robot and Human Interactive Communication (RoMan 2012) (pp. 718-724). IEEE Press. doi:10.1109/ROMAN.2012.6343836

Lu, a. S., Baranowski, J., Islam, N., \& Baranowski, T. (2012). How to engage children in self-administered 
dietary assessment programmes. Journal of Human Nutrition and Dietetics, 1-5. doi:10.1111/j.1365277X.2012.01258.x

Markus, H., Eichberg, J., \& Andre, E. (2012). Studies on grounding with gaze and pointing gestures in human-robot-interaction. In 4th International Conference on Social Robotics (ICSR 2012) (pp. 378387). Chengdu, China.

Meltzer, D. E. (2002). The relationship between mathematics preparation and conceptual learning gains in physics: A possible hidden variable in diagnostic pretest scores. American Journal of Physics, 70(12), 1259. doi:10.1119/1.1514215

Nalin, M., Baroni, I., Kruijff-Korbayova, I., Canamero, L., Lewis, M., Beck, A., ... Sanna, A. (2012). Children's adaptation in multi-session interaction. In Proceedings of the 21 st IEEE International Symposium in Robot and Human Interactive Communication (RoMan 2012).

Nalin, M., Baroni, I., Sanna, A., \& Pozzi, C. (2012). Robotic companion for diabetic children. In Proceedings of the 11th International Conference on Interaction Design and Children - IDC'12 (p. 260). New York, New York, USA: ACM Press. doi:10.1145/2307096.2307140

Nalin, M., Verga, M., Sanna, A., \& Saranummi, N. (2013). Directions for ICT research in disease prevention. In M. Cruz-Cunha, I. Miranda, \& P. Goncalves (Eds.), Handbook of research on ICTs for human-centered healthcare and social care services (pp. 229-247). Hershey, Pennsylvania, USA: IGI Global Press. doi: 10.4018/978-1-4666-3986-7

Office of Disease Prevention and Health Promotion. (2013). Physical activity guidelines. Website. Retrieved from http: / / www. health.gov/paguidelines

Richman, W. L., Kiesler, S., Weisband, S., \& Drasgow, F. (1999). A meta-analytic study of social desirability distortion in computer-administered questionnaires, traditional questionnaires, and interviews. Journal of Applied Psychology, 84(5), 754.

Riek, L. (2012). Wizard of Oz studies in HRI: A systematic review and new reporting guidelines. Journal of Human-Robot Interaction, 1(1), 119-136. doi:10.5898/JHRI.1.1.Riek

Ros, R., Baroni, I., \& Demiris, Y. (2014). Adaptive humanrobot interaction in sensorimotor task instruction: From human to robot dance tutors. Robotics and Autonomous Systems, 62(6), 707-720. doi: 10.1016/j.robot.2014.03.005

Ros, R., Coninx, A., Demiris, Y., Patsis, G., Enescu, V., \& Sahli, H. (2014). Behavioral accommodation towards a dance robot tutor. In Proceedings of the 2014 ACM/IEEE International Conference on Human-Robot Interaction - HRI'14 (pp. 278-279). New York, New York, USA: ACM Press. doi: $10.1145 / 2559636.2559821$

Ros, R., \& Demiris, Y. (2013). Creative dance: An approach for social interaction between robots and children. In A. A. Salah, H. Hung, O. Aran, \& H. Gunes (Eds.), Human behavior understanding (Lecture Notes in Computer Science) (Vol. 8212, pp. 40-51). Springer. doi:10.1007/978-3-319-02714-2_4

Ros, R., Nalin, M., Wood, R., Baxter, P., Looiije, R., Demiris, Y., .. Pozzi, C. (2011). Child-robot interaction in the wild: Advice to the aspiring experimenter. In Proceedings of the 13th International Conference on Multimodal Interfaces (pp. 335-342). Alicante, Spain: ACM Press.

Ryan, R. M., \& Deci, E. L. (2000). Self-determination theory and the facilitation of intrinsic motivation, social development, and well-being. American Psychologist, 55(1), 68-78. doi:10.1037/0003-066X.55.1.68

Saerbeck, M., Schut, T., Bartneck, C., \& Janse, M. D. (2010). Expressive robots in education: Varying the degree of social supportive behavior of a robotic tutor. In Proceedings of the SIGCHI Conference on Human Factors in Computing Systems (CHI 2010) (pp. 1613-1622). Atlanta, USA: ACM Press.

Salter, T., Werry, I., \& Michaud, F. (2007). Going into the wild in child-robot interaction studies. Intelligent Service Robotics, 1(2), 93-108.

Salter, T., Werry, I., \& Michaud, F. (2008). Going into the wild in childrobot interaction studies: Issues in social robotic development. Intelligent Service Robotics, 1(2), 93-108. doi:10.1007/s11370-007-0009-9

Schiefele, U. (1991). Interest, learning, and motivation. Educational Psychologist, 26(3-4), 299-323. doi: $10.1080 / 00461520.1991 .9653136$

Segura, E. M., Kriegel, M., Aylett, R., Deshmukh, A., \& Cramer, H. (2012). How do you like me in this: User embodiment preferences for companion agents. In Proceedings of the 12th International Conference on 
Intelligent Virtual Agents (Lecture Notes in Computer Science) (Vol. 7502, pp. 112-125). Santa Cruz, CA, USA: Springer Berlin / Heidelberg.

Shibata, T. (2011). Importance of physical interaction between human and robot for therapy. In C. Stephanidis (Ed.), Universal Access in Human-Computer Interaction: Applications and Services (Vol. 6768, pp. 437-447). Berlin, Heidelberg: Springer Berlin Heidelberg. doi:10.1007/978-3-642-21657-2

Short, E., Swift-spong, K., Greczek, J., Ramachandran, A., Litoiu, A., Grigore, E. C., . . Scassellati, B. (2014). How to train your dragonbot: Socially assistive robots for teaching children about nutrition through play. In 23rd IEEE Symposium on Robot and Human Interactive Communication (RoMan). Edinburgh, U.K.: IEEE Press.

Sidner, C. L. (2012). Engagement: Looking and not looking as evidence for disengagement. In Proceedings of the Workshop on Gaze in HRI (at HRI'12). Boston, MA, USA.

Sidner, C. L., Lee, C., Kidd, C. D., Lesh, N., \& Rich, C. (2005). Explorations in engagement for humans and robots. Artificial Intelligence, 166(1-2), 140-164. doi:10.1016/j.artint.2005.03.005

Stiehl, W. D., Lee, J. K., Breazeal, C., Nalin, M., Morandi, A., \& Sanna, A. (2009). The huggable: A platform for research in robotic companions for pediatric care. In Proceedings of the 8th International Conference on Interaction Design and Children - IDC'09 (p. 317). New York, New York, USA: ACM Press. doi: $10.1145 / 1551788.1551872$

Tanaka, F., Cicourel, A., \& Movellan, J. R. (2007). Socialization between toddlers and robots at an early childhood education center. In Proceedings of the National Academy of Sciences of the United States of America (Vol. 104, pp. 17954-8). doi:10.1073/pnas.0707769104

Tanaka, F., Movellan, J. R., Fortenberry, B., \& Aisaka, K. (2006). Daily HRI evaluation at a classroom environment: Reports from dance interaction experiments. In Proceedings of the 1st ACM SIGCHI/SIGART Conference on Human-Robot Interaction (HRI '06) (pp. 3-9). Salt Lake City, Utah, USA.

Tapus, A., Tapus, C., \& Matarić, M. J. (2008, February). Userrobot personality matching and assistive robot behavior adaptation for post-stroke rehabilitation therapy. Intelligent Service Robotics, 1(2), 169-183. doi:10.1007/s11370-008-0017-4

Tesser, F., Sommavilla, G., Paci, G., \& Cosi, P. (2013). Experiments with signal-driven symbolic prosody for statistical parametric speech synthesis. In Proceedings of the 8th ISCA Speech Synthesis Workshop (pp. 2-6). Barcelona, Spain.

Thill, S., Pop, C. A., Belpaeme, T., Ziemke, T., \& Vanderborght, B. (2012). Robot-assisted therapy for autism spectrum disorders with (partially) autonomous control: Challenges and outlook. Paladyn Journal of Behavioral Robotics, 3(4), 209-217.

Turkle, S., Breazeal, C., Dasté, O., \& Scassellati, B. (2006). Encounters with Kismet and Cog: Children respond to relational artifacts. In P. Messaris \& L. Humphreys (Eds.), Digital media: Transformations in human communication (pp. 1-20). New York: Peter Lang Publishing.

Wainer, J., Feil-Seifer, D. J., Shell, D. A., \& Mataric, M. J. (2007). Embodiment and human-robot interaction: A task-based perspective. In Proceedings of the 16th IEEE International Symposium on Robot and Human Interactive Communication (RoMan 2007) (pp. 872-877). IEEE. doi:10.1109/ROMAN.2007.4415207

Wang, W., Enescu, V., \& Sahli, H. (2013). Towards real-time continuous emotion recognition from body movements. Human Behavior Understanding, 8212, 1-11.

Wood, L. J., Dautenhahn, K., Rainer, A., Robins, B., Lehmann, H., \& Syrdal, D. S. (2013). Robot-mediated interviews-How effective is a humanoid robot as a tool for interviewing young children? PloS ONE, 8(3), e59448. doi:10.1371/journal.pone.0059448

World Health Organization. (2014). A healthy lifestyle. Website. Retrieved from http://www.euro.who.int/en/health-topics/disease-prevention/ nutrition/a-healthy-lifestyle

Primary authors' names and contact information: Alexandre Coninx, Personal Robotics Laboratory, Imperial College London, U.K. Email: a.coninx@imperial.ac.uk. Paul Baxter, Centre for Robotics and Neural Systems, The Cognition Institute, Plymouth University, U.K. Email: paul.baxter@plymouth.ac.uk. 


\section{APPENDIX A: QUESTIONNAIRES}

These questionnaires were administered to the children who participated in the proof-of-concept case study. The formatting has been changed: the originals used emoticons in the Likert-scale responses to support the sentiment of the possible responses. The original questionnaires were administered in Italian; what follows are translations of these. See main body of text for details.

\section{A1. Knowledge Questionnaire}

1 How often should we eat fruit and vegetables?

2

3 Carbohydrates are in:
(a) Meat
(b) Bread
(c) Apple
(d) Jam

4

5

6

7

8

9
Choose the correct sequence for fruit, from most carbohydrates to least

Choose the correct sequence for vegetables, from most carbohydrates to least Choose the correct answer: the first food contains carbohydrates, the second proteins, and the third fats Choose the correct answer: the first food is a cereal, the second a legume, and the third a vegetable

What kinds of nutrients are contained in foods?

Which drink contains the most carbohydrates? Which of the following contains 15 grams of simple sugar? If you are going on a school trip and have to walk around the city all day, which of the following is NOT necessary to bring? ["every day, 5 portions vegetables, 3 of fruit", "every day, 5 portions vegetables, 5 of fruit", "once a day", "only when I want to"] ["swimming-walking-watching TV-lifting weights", "walking-swimming-lifting weights-watching TV", "watching TV-lifting weights-swimming-walking"]

[true, false]

[true, false]

[true, false]

[true, false]

["banana-apple-peach", "peach-banana-apple”, "apple-peach-banana”, “don't know”]

["potatoes-carrots-courgette", "carrots-courgette-potatoes", "courgette-potatoes-carrots", “don't know”] ["chicken-butter-potatoes", "potatoes-chicken-butter", "butter-potatoes-chicken", "don't know"] ["corn-beans-courgette", "beans-courgette-corn", "courgette-corn-beans", "don't know"] ["carbohydrates and fibre", "proteins and vitamins", "fats and minerals", "carbohydrates, proteins, fats, vitamins and minerals", "don't know"]

[milk, soda, juice, orange juice]

["6 bags of sugar", "a can of soda", "a sugary fruit juice", "3 sugary sweets"]

[insulin pen/pump, glucometer, corrections, urine stick, test strips, sterile gloves] 


\section{A2. Habits Questionnaire}

1 How many portions of vegetables do you eat during the day?

2 How many portions of fruit do you eat during the day?

3 What drink do you mostly have during the day?

$4 \quad$ Do you do any sport?

(a) If yes, what?

5

What do you usually do in your free time?

6

Do you constantly check your glycaemia?

7 How do you usually correct hypoglycaemia?

(a) number of ---- sugar bags

(b) number of _---- sweets

(c) number of _--- sweetened sodas

(d) how much sweetened orange juice do you drink?

(e) Other

8

Are you able to do carbohydrates counting?
[“I do not eat", 1, 2, 3, 4, 5]

["I do not eat", 1, 2, 3, 4, 5]

["milk”, "soda", “juice”, “orange juice”, “water”, "cold or hot tea"]

[yes, no]

free text

["I go to the park", "Walking the dog", "Do some sport", "I watch TV", "I play videogames on computer or mobile", "I tidy my room", "Other (specify)"]

["Yes, regularly, before meals and two hours after", "No, I check it only when it is high or low", "I check only when my parents tell me to"] choose one of the following

number entry

number entry

number entry

number entry

free text

["Yes, I do it routinely", "No, I have not yet learned how to do it", "Yes, with the help of my parents"] 


\section{A3. Engagement Questionnaire}

$1 \quad$ How much did you like Nao?

$2 \quad$ What did you like most about Nao?

3 Would you like to play with Nao again?

$4 \quad$ Why would you like to (or not)?

5 What do you think of Nao? Nao is like a...

6 Did you completely understand what Nao said?

$7 \quad$ How much fun was the QUIZ game you played with Nao?

8 How much fun was the DANCE game you played with Nao? How much fun was the SORTING

9

10 game you played with Nao? Have you learned NEW things from Nao?

Would you like to learn something more with Nao?

12 If yes, what would you like to learn with Nao? From the following words, which describes Nao best?

14 In your opinion, Nao was...

15 [not at all, not so much, so so, a lot, very much] free text

[yes, no, maybe]

free text

[friend, pet, adult, toy, computer]

[yes, no, sometimes]

[not at all, not so much, so so, a lot, very much]

[not at all, not so much, so so, a lot, very much]

[not at all, not so much, so so, a lot, very much]

[yes, no, maybe]

[yes, no, maybe]

free text

[mechanical, nice, funny, smart, fake, fragile, tender, affectionate, boring, scientific]

[quite happy, quite sad, neutral, Nao has no feeling, don't know]

[yes it can recognise emotions, yes it can recognise and understand emotions, no, don't know] 